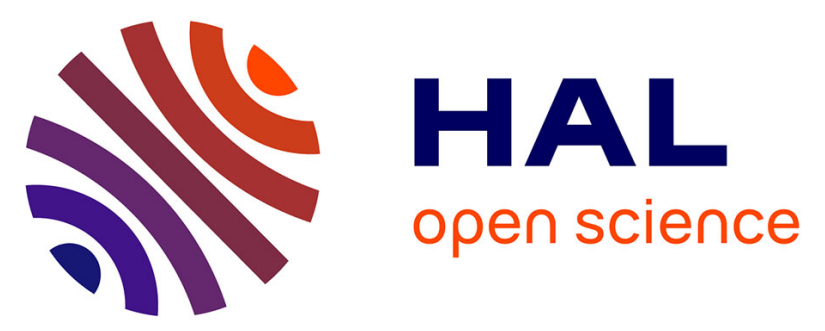

\title{
A first assessment of the strength of cometary particles collected in-situ by the COSIMA instrument onboard ROSETTA
}

Klaus Hornung, Sihane Merouane, Martin Hilchenbach, Yves Langevin, Eva Maria Mellado, Vincenzo Della Corte, Jochen Kissel, Cecile Engrand, Rita Schulz, Jouni Ryno, et al.

\section{To cite this version:}

Klaus Hornung, Sihane Merouane, Martin Hilchenbach, Yves Langevin, Eva Maria Mellado, et al.. A first assessment of the strength of cometary particles collected in-situ by the COSIMA instrument onboard ROSETTA. Planetary and Space Science, 2016, 133, pp.63-75. 10.1016/j.pss.2016.07.003 . insu-01351370

\section{HAL Id: insu-01351370 \\ https://hal-insu.archives-ouvertes.fr/insu-01351370}

Submitted on 3 Oct 2016

HAL is a multi-disciplinary open access archive for the deposit and dissemination of scientific research documents, whether they are published or not. The documents may come from teaching and research institutions in France or abroad, or from public or private research centers.
L'archive ouverte pluridisciplinaire HAL, est destinée au dépôt et à la diffusion de documents scientifiques de niveau recherche, publiés ou non, émanant des établissements d'enseignement et de recherche français ou étrangers, des laboratoires publics ou privés. 


\section{Author's Accepted Manuscript}

A first assessment of the strength of cometary particles collected in-situ by the COSIMA instrument onboard ROSETTA

Klaus Hornung, Sihane Merouane, Martin Hilchenbach, Yves Langevin, Eva Maria Mellado, Vincenzo Della Corte, Jochen Kissel, Cecile Engrand, Rita Schulz, Jouni Ryno, Johan Silen, the

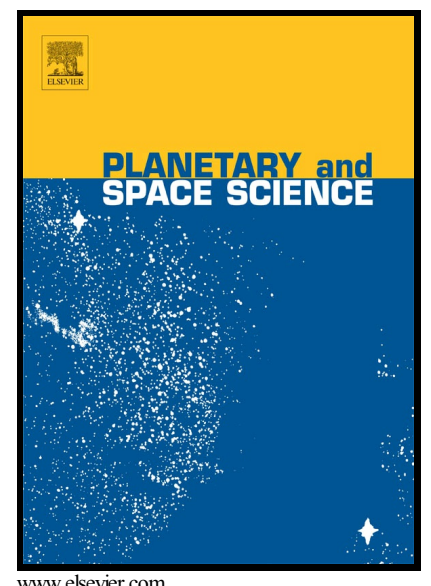
COSIMA team

PII: $\quad$ S0032-0633(15)30095-7

DOI: $\quad$ http://dx.doi.org/10.1016/j.pss.2016.07.003

Reference: PSS4203

To appear in: Planetary and Space Science

Received date: 9 November 2015

Revised date: 9 July 2016

Accepted date: 14 July 2016

Cite this article as: Klaus Hornung, Sihane Merouane, Martin Hilchenbach, Yve: Langevin, Eva Maria Mellado, Vincenzo Della Corte, Jochen Kissel, Cecil Engrand, Rita Schulz, Jouni Ryno, Johan Silen and the COSIMA team, A firs assessment of the strength of cometary particles collected in-situ by the COSIM instrument onboard ROSETTA, Planetary and Space Science http://dx.doi.org/10.1016/j.pss.2016.07.003

This is a PDF file of an unedited manuscript that has been accepted fo publication. As a service to our customers we are providing this early version o the manuscript. The manuscript will undergo copyediting, typesetting, an review of the resulting galley proof before it is published in its final citable form Please note that during the production process errors may be discovered whic could affect the content, and all legal disclaimers that apply to the journal pertain 
2 nd revision: July 09, 2016

\title{
A first assessment of the strength of cometary particles collected in-situ by the COSIMA instrument onboard ROSETTA
}

Klaus Hornung ${ }^{1}$, Sihane Merouane ${ }^{2}$, Martin Hilchenbach ${ }^{2}$, Yves Langevin ${ }^{3}$, Eva Maria Mellado ${ }^{1}$, Vincenzo Della Corte ${ }^{4}$, Jochen Kissel ${ }^{2}$, Cecile Engrand $^{5}$, Rita Schulz ${ }^{6}$, Jouni Ryno ${ }^{7}$ Johan Silen $^{7}$ and the COSIMA team

(1) Universität der Bundeswehr München, LRT-7, 85577 Neubiberg, Germany, klaus.hornung@unibw.de

(2) Max-Planck-Institut für Sonnensystemforschung, Justus-von-Liebig-Weg 3, 37077 Göttingen, Germany.

(3) Institut d'Astrophysique Spatiale, CNRS / Université Paris Sud, Bâtiment 121, 91405 Orsay, France.

(4) INAF - Instituto di Astrofisica e Planetologia Spaziali, Via Fosso del Cavaliere, 100, 0133, Rome, Italy.

(5) Centre de Sciences Nucléaires et de Sciences de la Matière, Bat.104, 91405 OrsayCampus,France.

(6) European Space Agency, Scientific Support Office, Keplerlaan 1, Postbus 299, 2200 AG Noordwijk, The Netherlands.

(7) Finnish Meteorological Institute, Climate Research, Erik Palmenin aukio 1, P.O.Box 503, FI00101 Helsinki, Finland.

\begin{abstract}
:
Soon after the arrival of the ROSETTA spacecraft at Comet 67/P Churyumov-Gerasimenko the onboard instrument COSIMA ("Cometary Secondary Ion Mass Analyzer") collected a large number of cometary dust particles on targets from gold black of thickness between 10 and $30 \mu \mathrm{m}$. Inspection by its camera subsystem revealed that many of them consist of smaller units of typically some tens of micrometers in size. The collection process left the smaller dust particles in an essentially unaltered state whereas most particles larger than about $100 \mu \mathrm{m}$ got fragmented into smaller pieces. Using the observed fragment size distributions, the present paper includes a first assessment of the strength for those dust particles that were disrupted upon impact.
\end{abstract}

Keywords: Cometary dust, Mechanical strength, Impact fragmentation, Rosetta mission. 


\section{Introduction}

Interplanetary and cometary dust particles have been collected in the past in various ways where the corresponding decelerations span a vast range of about 12 orders of magnitude. The softest method is by deceleration in the Earth's atmosphere (from several $10 \mathrm{~km} / \mathrm{s}$ within several $10 \mathrm{~km}$ ) resulting in several $10^{3} \mathrm{~m} / \mathrm{s}^{2}$ and some of these particles got to the Earth's surface essentially unaltered (Duprat et al., 2007, Engrand et al., 2015). Within the STARDUST aerogel collection, deceleration was from 6.1 $\mathrm{km} / \mathrm{s}$ within several centimeters target thickness, leading to an order of $10^{9} \mathrm{~m} / \mathrm{s}^{2}$, enough to substantially change their shape, but still retaining some of them in solid state (Brownlee 2014). Hypervelocity impact onto a compact silver target within the STARDUST/CIDA instrument produced an order of $10^{13} \mathrm{~m} / \mathrm{s}^{2}$ enough to vaporize and partially ionize the dust (Kissel et al., 2003). Finally the most extreme setting of hypervelocity impact within the PUMA and PIA instruments onboard the Halley missions (from $80 \mathrm{~km} / \mathrm{s}$ within the dust's size of $\mu \mathrm{m}$ or even less) resulted in values of up to several $10^{15} \mathrm{~m} / \mathrm{s}^{2}$ leading to complete vaporization of the collected dust and to a substantial degree of single ionization.

The rendezvous phase of ROSETTA near the nucleus of comet 67P/Churyumov-Gerasimenko made it possible, for the first time, to collect cometary dust at very low speeds $(<10 \mathrm{~m} / \mathrm{s}$, Rotundi et al., 2015). The decelerations of particles on the COSIMA targets were in the range of at most $10^{6} \mathrm{~m} / \mathrm{s}^{2}$, just enough to cause mechanical damage upon impact. The present contribution makes an attempt to derive information on the mechanical properties, such as strength, of the incoming dust from an analysis of the observed damages.

\section{Observational data}

Since August 2014 the COSIMA instrument continuously exposed its targets to collect cometary dust particles in the coma of Comet 67P/Churyumov-Gerasimenko. The targets consisted of metal plates of $1 \mathrm{x} 1 \mathrm{~cm}^{2}$ from gold, covered by a layer $(10$ to $30 \mu \mathrm{m})$ of gold black, which is a highly porous aggregate of nanometer sized building blocks (mean density about $2 \mathrm{~g} / \mathrm{cm}^{3}$ ) sticking together by adhesive forces. The metal black layer was chosen to decelerate and fixate the incoming dust for later chemical analysis by secondary ion mass spectrometry (Kissel et al., 2009). The COSISCOPE microscope, a subsystem of COSIMA has been designed to locate captured dust particles for the correct positioning of the analyzing ion beam. However, already after a few weeks of COSIMA operation, the images showed a large number of collected dust particles with an extraordinary wealth of morphological details and soon it became clear that those details have their independent scientific value. A first discussion is contained in a recent report (Langevin et al., 2016). Many of the observed patterns showed clear signs of impact generated fragmentation, an observation which can be linked to the mechanical strength of the incoming dust particle.

We report on data obtained during the first half year of data acquisition, from Aug. 2014 to Feb. 2015. In this period ROSETTA was very close to the comet (down to $10 \mathrm{~km}$ ) and COSIMA collected and identified a total of about 7500 dust particles on two of its target assemblies, D0 and CF, each equipped with 3 gold black targets (named 1D0,2D0,3D0 and 1CF,2CF,3CF). COSISCOPE images have been produced with a spatial resolution of about $10 \mu \mathrm{m}$, which was achieved by combining 4 individual $14 \mu \mathrm{m}$ pixel-size images (each containing 1024x1024 values) shifted by half a pixel in the $\mathrm{X}$ or Y direction. This was possible because COSIMA can translate its targets with very high precision. Illumination was under grazing incidence. On all images displayed in the present report illumination is from one side only (right) in order to provide a better recognition of the threedimensional situation by showing clear shadows. However the data set also contains images with illumination from left side and we included these in the quantitative data reduction below. All images shown are in log-scale, i.e. the grey level of a pixel corresponds to the logarithm of the value recorded by the camera. For more details of the experimental situation we refer to the report of Langevin et al. 


\section{ACCEPTED MANUSCRIPT}

(2016). For easier reference the collected particles were given individual names as well as a last name according to the time period of collection.

\subsection{Overview of the whole data set}

Fig. 1a shows a part of target $2 \mathrm{CF}$. It gives an impression of the diversity of breakup situations. Big and medium sized impact clusters, and such with only a few fragments can be seen. The overwhelming number of particles is small and appears to be undamaged. In this paper we will use "cluster" for the structured objects on our targets (see also Langevin et al. 2016). The term "agglomerate" will be used for a structured dust particle before its impact. In the image the gold black background looks rather smooth at the COSISCOPE resolution of $\sim 10 \mu \mathrm{m}$. A closer look with electron microscopy (see Fig. 5 below), however, shows surface roughness in the order of several $\mu \mathrm{m}$. The radial distribution of fragments up to a distance from the impact center, where the mean area number density of all particles is reached, was determined, in order to find out which of them belong to a certain impact event. This gives the outer boundary of the cluster. An example will be discussed in detail in Fig. 2c for the impact event on the right-hand side of Fig. 1a marked by an arrow. There are also long shadows from which height information can be derived.

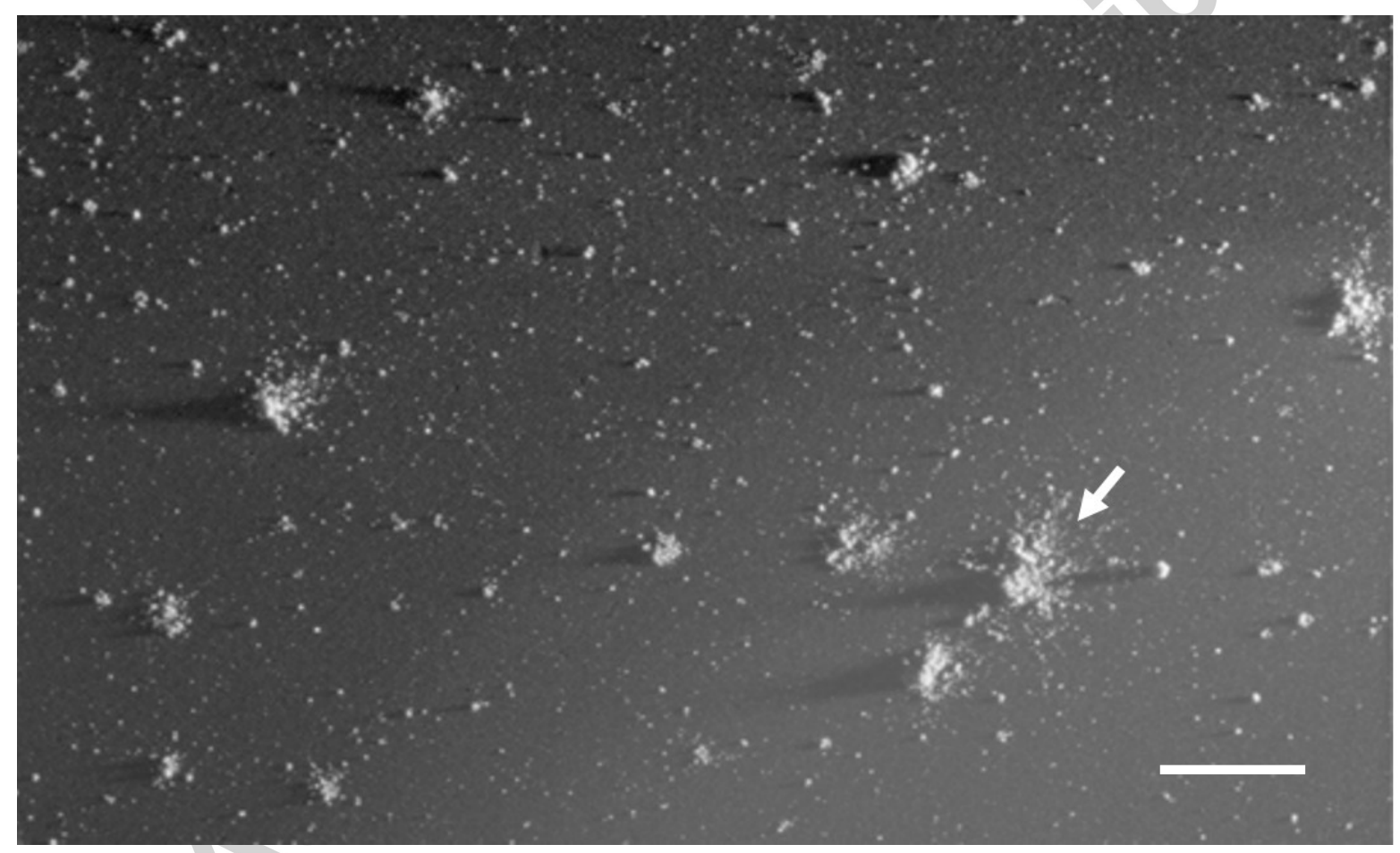

Fig. 1a: A part of target 2CF, status Feb 10, 2015, (scale bar: $1 \mathrm{~mm}$ ).

Fig. 1b shows the size-frequency distribution of the 7524 particles of the present data set. Size binning is in steps of $10 \mu \mathrm{m}$. The sizes of the particles (diameters $d$ ) were derived from the apparent area $a$ in the image plane as well as from information on their height $h$ using the shadows cast by them: $d=\left(\frac{6}{\pi} \cdot a \cdot h \cdot \varepsilon\right)^{1 / 3} \quad$ where $a \cdot h \cdot \varepsilon$ is the volume. For the bigger impact clusters the surrounding fragments, which are recognized as belonging to the cluster, are contributing to the value of $a$. By this $d$ is defined as the "equivalent sphere diameter" of the incoming dust particle. Due to the impact damage, we cannot reconstruct the real shape of the incoming dust particle. $\varepsilon$ is a geometry factor for the particle after its impact. It is $=1 / 3$ for a pyramidal shape, $=1 / 2$ for a saddleback-roof shape, $=2 / 3$ for a 


\section{ACCEPTED MANUSCRIPT}

spherical shape, and $=1$ for a cylindrical shape. In Fig. 1 a one finds examples for each case. The shadow of the big round particle in Fig. 1a next to the one marked by an arrow, e.g. shows a nice elliptical shape suggesting a spherical particle, obviously undamaged, thus has an $\varepsilon$ value close to $2 / 3$, whereas for the big clusters we derive shapes suggesting values between $1 / 3$ and $1 / 2$. A few flat clusters show shapes pointing to $\varepsilon \approx 1$. For simplicity, we take a common value of $\varepsilon=1 / 2$ for all particles. From this choice a possible error in $d$ is small, since $\varepsilon$ enters only with power of $1 / 3$ into the equation. Only for some rare cases of a cylindrical shape it reaches $20 \%$, but for pyramidal and spherical shapes it is 14 and $9 \%$ respectively. A common value for all particles has the advantage to keep a clear relationship between diameter and volume $V=(a \cdot h \cdot \varepsilon)$ from the present area and height data. Height values $h$ are available for the larger particles and we take the values listed in the previous report of Langevin et al. (2016) for our data evaluation. The remaining smaller particles appear mostly undamaged. As the optical resolution does not allow the determination of their real shape, we treat them as spheres, and we take as height the diameter of a circle having the same area as the apparent area in the image plane: $h=\sqrt{\frac{4}{\pi} \cdot a}$. We also take $\varepsilon=1 / 2$ for these cases. The resulting equivalent sphere diameters $d$ are in the range of 15 to 300 $\mu \mathrm{m}$. Fitting the distribution of Fig. $1 \mathrm{~b}$ with a power law, results in an index of $-3.3 \pm 0.2$. We have chosen for Fig. $1 \mathrm{~b}$ the frequency distribution of the sizes rather than the cumulative distribution to be consistent with the size distribution of the fragments Fig. 2d, which we need in the frequency-form as an input to later, theoretical discussions (Sec. 3). The distribution of the sizes will be discussed in more detail in a forthcoming paper (Merouane et al., 2016).

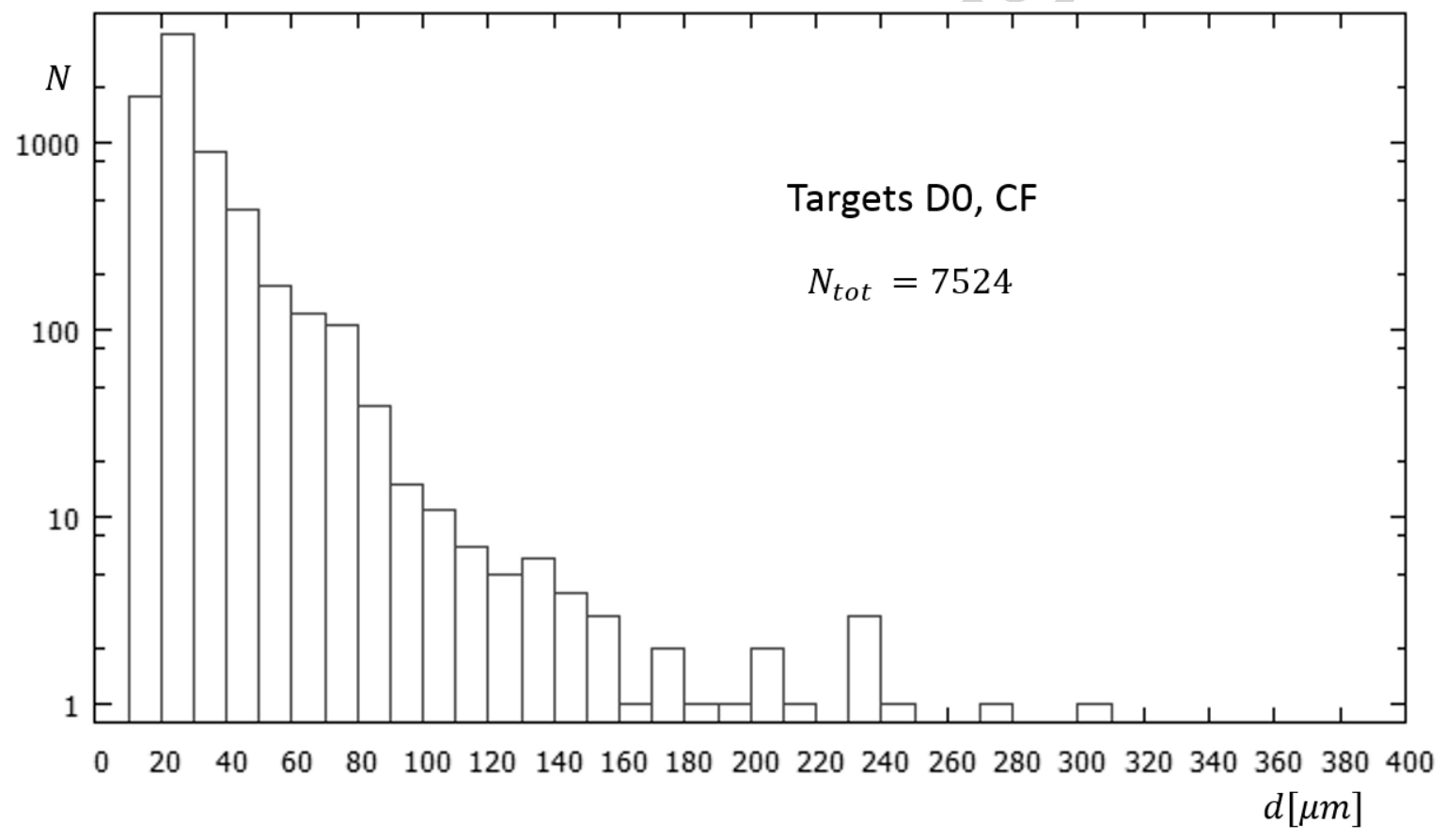

Fig. 1b: Frequency distribution of the particle sizes for the collection period from Aug. 2014 to Feb. 2015. $d$ is the equivalent sphere diameter $d=\left(\frac{6}{\pi} \cdot a \cdot h \cdot \varepsilon\right)^{1 / 3}, N=$ number per $10 \mu m$ diameter interval, $a=$ area in image plane, $h=$ particle height, $\varepsilon=$ geometry factor $(\varepsilon \approx 0.5$ assumed, see text).

2.2 Diversity of the phenomena for selected particles 


\section{ACCEPTED MANUSCRIPT}

We used enlarged images of selected areas on the targets for further analyses. On these images (Figures 2 below) most of the particles present themselves as cluster-like structures, consisting of individual sub-units in the order of some tens of $\mu \mathrm{m}$ in size, reminiscent of a "bunch of grapes" (see Figures 2a, 2b below). We shall denote these sub-units as "cluster elements" or simply "elements" in the remainder of the paper. If a particle disintegrated upon impact, the observed debris will be denoted as "fragments". The elements as well as the fragments may consist of even smaller units down to submicron sizes (a size range, which is accessible to the MIDAS instrument onboard ROSETTA, Riedler et al., 2007, Bentley et al., 2014). Interplanetary dust particles (IDP's) collected in the upper atmosphere also show such fine structures (Brownlee, 1985). Another result is that the collected particles do not penetrate much into the black layer, as had been observed in the laboratory for higher speeds (Hornung et al, 2014). Some particles stick out of the targets, and the contact area with the black substrate is small in relation to their height, as shown in Langevin et al. (2016). This can only be, if the impact speeds were very low, much lower than expected. From our laboratory experience we estimate values of a few $\mathrm{m} / \mathrm{s}$. From the images alone, however, a realistic value cannot be established. Confirmation came from the GIADA instrument onboard ROSETTA, which measured speeds directly, and found values of less than $10 \mathrm{~m} / \mathrm{s}$ (Rotundi et al., 2015, Della Corte, 2015).
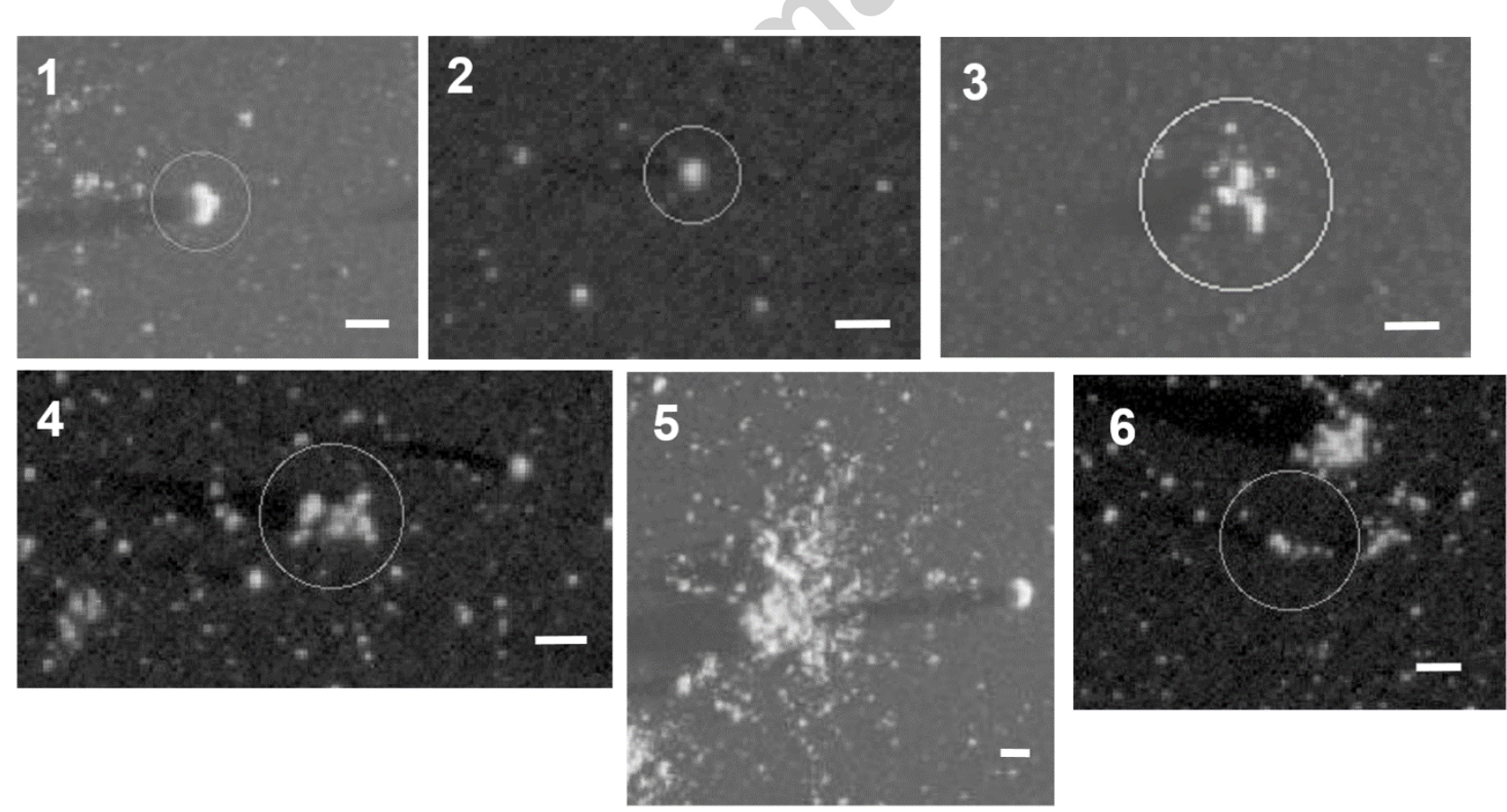

Fig. 2a: 1 : no breakup: 2CF Lambert Kolima.3, $\mathrm{d} \approx 108 \mu \mathrm{m}, \mathrm{h} \approx 115 \mu \mathrm{m}$.

2: no breakup: $1 \mathrm{CF}$ Pecine Ala Kitka, $\mathrm{d} \approx 41 \mu \mathrm{m}, \mathrm{h} \approx 45 \mu \mathrm{m}$.

3: simple breakup: 2D0 Stefanie Saimaa, $\mathrm{d} \approx 87 \mu \mathrm{m}, \mathrm{h} \approx 45 \mu \mathrm{m}$, fragment sizes 15-40 $\mu \mathrm{m}$ (diameter).

4: simple breakup: 2CF Pertti Kolima.3, $\mathrm{d} \approx 89 \mu \mathrm{m}, \mathrm{h} \approx 40 \mu \mathrm{m}$, fragment sizes 20-35 $\mu \mathrm{m}$.

5: catastrophic breakup: 2CF Nilda Kolima.3, $\mathrm{d} \approx 309 \mu \mathrm{m}, \mathrm{h} \approx 140 \mu \mathrm{m}$, fragment sizes 10-35 $\mu \mathrm{m}$

5 (the compact spherical particle to the right is Lambert, see panel 1 and is not part of Nilda).

6: shedding upon rolling: $2 \mathrm{CF}$ Clarence Kolima.3, $\mathrm{d} \approx 63 \mu \mathrm{m}, \mathrm{h} \approx 68 \mu \mathrm{m}$, fragment sizes 15-20 $\mu \mathrm{m}$.

$d=\left(\frac{6}{\pi} \cdot a \cdot h \cdot \varepsilon\right)^{1 / 3}, \mathrm{a}=$ area in image plane, $\mathrm{h}=$ height as derived from shadow, $\varepsilon=$ geometry factor 


\section{ACCEPTED MANUSCRIPT}
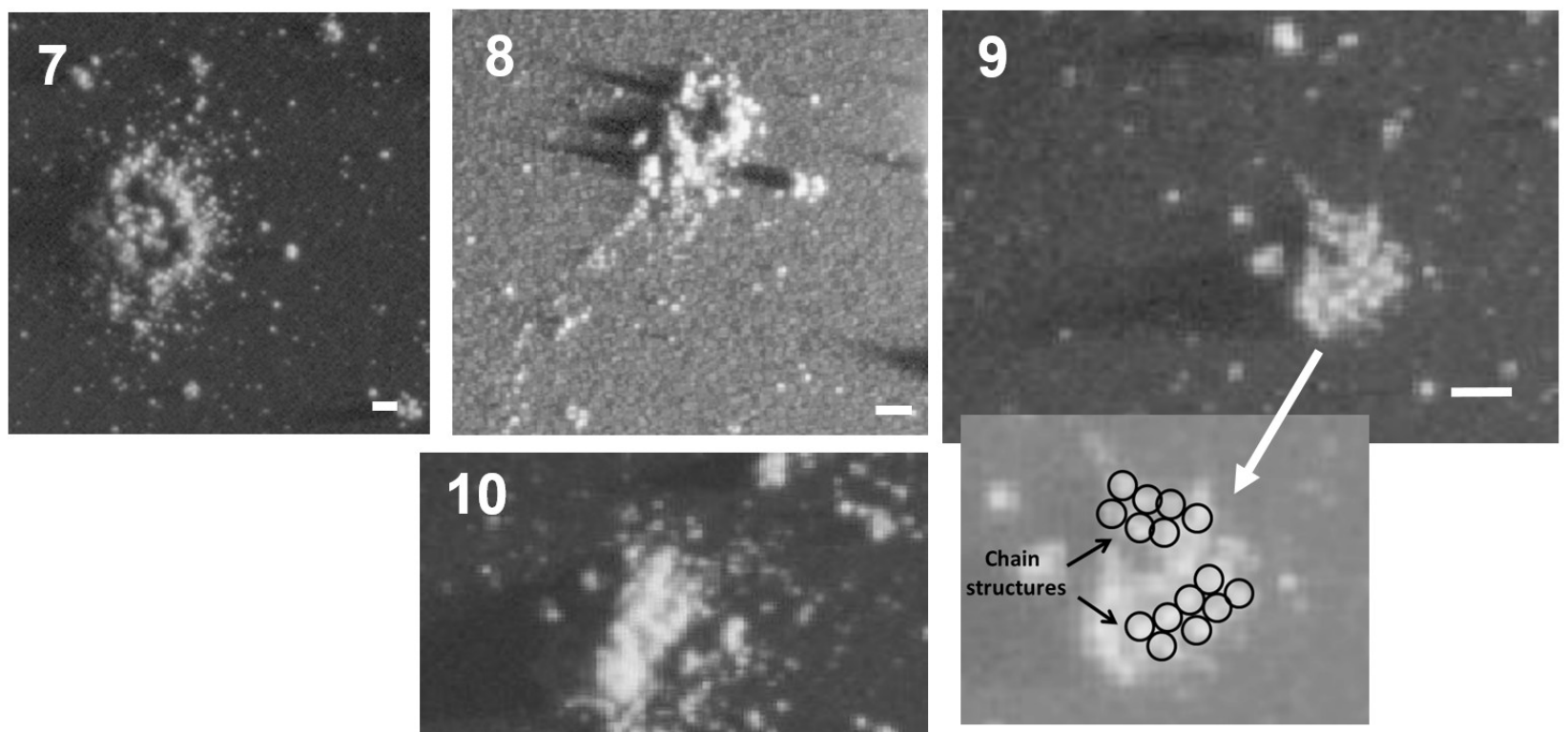

Fig. 2b: $\quad 7$ : depression at center: 2CF Jessica Lummene.2, $\mathrm{d} \approx 207 \mu \mathrm{m}, \mathrm{h} \approx 40 \mu \mathrm{m}$, element sizes 15-40 $\mu \mathrm{m}$.

8: depression at center: 3D0 Kamil Ukonvesi, $\mathrm{d} \approx 179 \mu \mathrm{m}, \mathrm{h} \approx 50 \mu \mathrm{m}$, element sizes 15-30 $\mu \mathrm{m}$.

9: linear chains of elements: $2 \mathrm{CF}$ Adeline Kolima.3, $\mathrm{d} \approx 120 \mu \mathrm{m}, \mathrm{h} \approx 50 \mu \mathrm{m}$, element sizes $\approx 20 \mu \mathrm{m}$.

10: shedding from a stronger core: $2 \mathrm{CF}$ Jean-Baptiste Kolima.3, $\mathrm{d} \approx 214 \mu \mathrm{m}, \mathrm{h} \approx 100 \mu \mathrm{m}$, element sizes $\approx 15-40 \mu \mathrm{m}$.

All scale bars $100 \mu m$. 
Depending on the degree of impact damage, we categorize the breakup events into 3 groups: a) "no breakup". b) "simple breakup", i.e. breakup into a few, smaller fragments, remainig close together, c) "catastrophic breakup" into many fragments, much smaller than the parent, in the neighbourhood, and with a pile of fragments as remnant at the center. In many cases the largest particles are of type c). An example is Nilda, Fig. 2a-5. Intermediate particles belong mostly to type b). Examples are Stefanie and Pertti (Fig. 2a-3, 2a-4). Pertti does show some fragments in the neighbourhood, which puts it closer to c), however. Type a), no breakup, seems to occur for small particles although it becomes increasingly difficult to define fragmentation when particles are imaged with only a few pixels. It is still possible down to $\sim 40-50 \mu \mathrm{m}, 4$ Cosiscope pixels, or 5 resolved elements with sub-pixel sampling. Pecine (Fig. 2a-2) is an example of such a particle, which did not fragment given its well defined shadow.

There are also cases where large dust particles do not fragment, like Lambert (Fig. 2a-1), being the best candidates for large, compact dust. These particles should be less porous than other large dust particles and they should have a higher strength (Rotundi et al., 2015, Langevin et al. 2016). For large particles we also observe mixed types of partial fragmentation (Fig. 2b 7-10). We shall discuss these in Sec. 2.2.3 where we denote them as "damaged". Both types b) and c) fulfill the conventional criterion for breakup, according to which the largest fragment mass has to be smaller than $1 / 2$ of the total particle mass. In an early work Hartmann (1978) showed that the transition from no damage to complete fragmentation occurs within a short range of impact speeds. For dirt clods consisting of glued-together silicate particles, this transition is observed between 1 and $2 \mathrm{~m} / \mathrm{s}$. The absence of a larger transition range is of relevance for our discussion of strength, when we use speed as a parameter (Sec. 3.1).

For the determination of strength from fragment sizes (Sec. 3.3), their lower and upper limits are of importance. These numbers are included in the figure captions of Figs. $2 \mathrm{a}$ and $2 \mathrm{~b}$, and summarized in Table 1. The basic observation is, that the size range of fragments is very narrow. Most of them are between 15 and $40 \mu \mathrm{m}$. Even when there is no fragmentation of the entire particle, its inner structure is clearly visible in most cases. Therefore we also give ranges for the size of these inner structures of the particles in Fig. 2b 7-10. They are not the debris of a captured solid, but rather elements making up the particle.

\subsubsection{Examples for spatial and size distributions of fragments}

The impact fragmentation sites are discovered by visual inspection of resolution-enhanced images. However, once spotted, they require further analyses. For big impact clusters e.g. it is interesting to find out, which particles are parts of the fragment halo, and which arrived independently as "singles". See 2 CF Nilda, Fig. 2a-5 as an example. The positions of the fragments around the central pile are shown in Fig. 2c left. Next, the location is calculated, for which the sum of the distances to all points is a minimum. It turns out, that this point lies within the central part of the pile, and is marked in the plot with a full circle. We take this point as "source center" for the radial emission of fragments into its surroundings. The right part of Fig. 2c shows the radial distribution of the fragments vs. the distance $r_{d}$ from this source center. It is binned in rings of $100 \mu \mathrm{m}$ width, and reaches a maximum at $\sim 350 \mu \mathrm{m}$, then drops to very low values at $\sim 750 \mu \mathrm{m}$. The outer boundary of the fragment cloud is then defined as the distance $r_{d}$ where the area number density within the ring reaches ambient values (in the present case about 20 part $/ \mathrm{mm}^{2}$ at $r_{d}=750 \mu \mathrm{m}$ ). This means that within $400 \mu \mathrm{m}$ the number of fragments drops to $\sim$ zero, and within $\sim 200 \mu \mathrm{m}$ to half of its maximum value. We define this half length as the characteristic travel length $l_{t}$ of the outward movement of the fragments. One plausible kind of the outward movement is rolling $\left(l_{t}=l_{R} \approx 200 \mu \mathrm{m}\right)$. We shall show later (Sec. 3.2) that this length is closely related to the sticking forces between dust and surface, and that it depends on both, fragment size, and impact speed. In theoretical models of dust agglomeration the rolling 


\section{ACCEPTED MANUSCRIPT}

movement is considered to be an important intergrain dissipation mechanism (see e.g. Kimura, 2015). From Fig. 2c it follows, that the fraction of "singles" in the fragment halo is small enough, so we can take the fragment sizes from an inspection of the entire halo.

The simple breakup of dust particles of $\sim 50-100 \mu \mathrm{m}$ can be detected visually as the fragments remain much closer to the impact site. For quantitative results we compare two lengths: (1) the mean distance between fragments within the broken particle, and (2) the mean value $l_{d}$ of interparticle distances (next neighbour) in a larger area around the impact, usually up to 10 times the broken particle's diameter. For the examples in Fig. 2 the results are: 2CF Pertti $l_{d} \approx 200 \mu m$, and 2D0 Stefanie $l_{d} \approx 600 \mu \mathrm{m}$. The distances between the fragments are each less than the equivalent sphere diameter of the dust particle, $<80 \mu \mathrm{m}$ in both cases. Again the contribution from background "singles" is very small at the impact location.
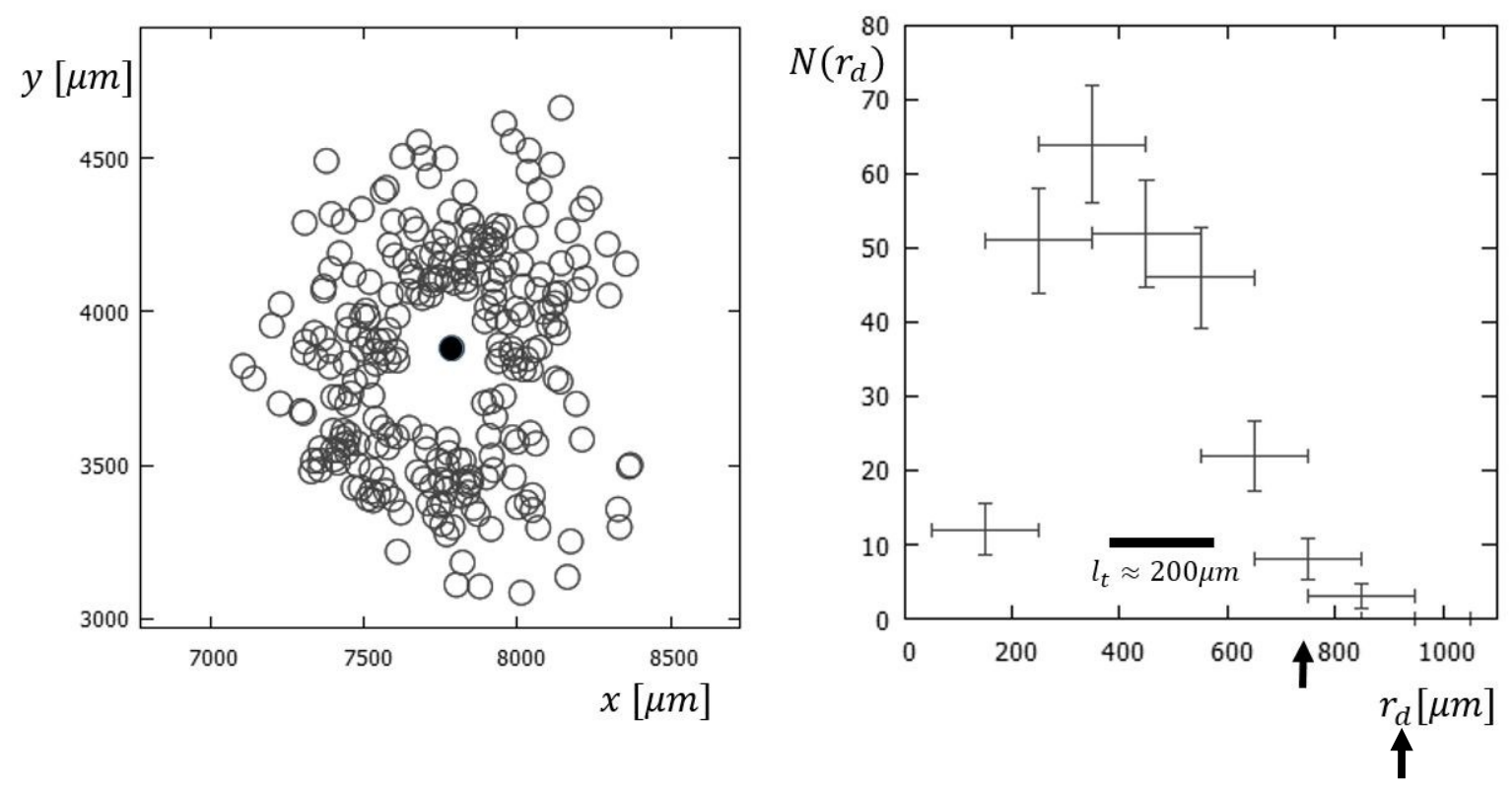

Fig. 2c: Fragment spatial distribution around 2CF Nilda Kolima.3 (see Fig. 2a-5). Left: Positions of fragments on target $2 \mathrm{CF}$. Zero point of coordinate system is the lower left corner of the $1 \mathrm{x} 1 \mathrm{~cm}^{2}$ target plate (see Langevin et al. 2016). The full central circle marks the fragment's "source center" from which the sum of distances to all fragments is a minimum. Right: Number of fragments within a ring of $100 \mu \mathrm{m}$ width as a function of radial distance from the source center (horizontal error bars are $\pm 100 \mu m$; vertical error bars: $\pm \sqrt{N}) . l_{t}$ : characteristic travel length of the fragments, defined by $N$ dropping to half of its maximum value. The arrow indicates where the mean ambient particle area number density of about $20 / \mathrm{mm}^{2}$ is reached.

For the discussion of strength, see Sec. 3, the size distribution of fragments is of interest for the types $\mathrm{b}$ and c, i.e. simple and catastrophic breakup. Fig. $2 \mathrm{~d}$ shows the example of $2 \mathrm{CF}$ Nilda Kolima.3. Fragments are contained in the surroundings of the central rubble pile up to the cloud limit at about $750 \mu \mathrm{m}$. It shows the size frequency because we'll need it later for an integration of forces over the size distribution. The result for the slope is $-3.9 \pm 0.5$. Due to uncertainties in size (horizontal error bars $\pm 25 \%$ ) and in number (vertical error bar $\pm \sqrt{N}$ ) this result has more uncertainties. We will show, however, that the final result for strength depends only insensitively on this numerical value. When we treat 2D0 Stefanie Saimaa and 2CF Pertti together for better statistics, we get a value of - 
2.3. In Sec. 3 we will use a common value of -3.0 and the individual values for Nilda, Stefanie and Pertti.

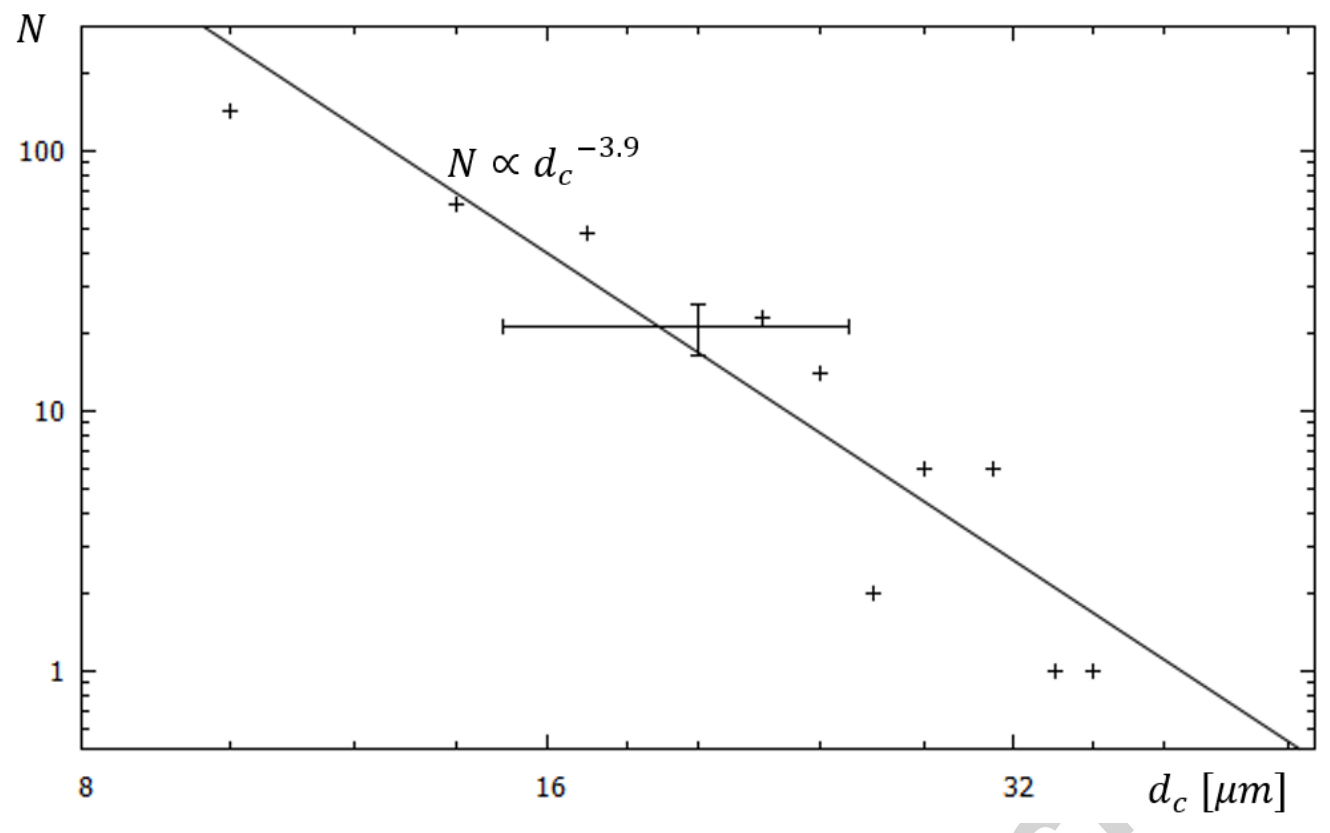

Fig. 2d: Frequency distribution of fragment sizes for the example of Fig. 2c (2CF Nilda Kolima.3).

\subsubsection{Special observations}

Another phenomenon, namely shedding, (Clark et al., 2004), shows up in some cases. An example is Jean-Baptiste, Fig. 2b-10. The image shows a central part looking compact, surrounded by small fragments. It may be explained by a stronger core, which has some loose bits sitting at its surface being detached by the impact, while the central part is intact. A mechanism for transferring energy to the loosely bound bits could be elastic waves through the central part generated by the impact (see Wurm et al. 2005).

For large particles, in case of complete (Fig. 2a-5), but also of partial fragmentation (Figures 2b$7,8,10)$, movement of fragments along the target surface is observed, probably in a kind of rolling movement. The rotation could be generated by two mechanisms: 1.The incoming dust has touched the inclined walls of the collimating entry funnel in front of the target assembly, such transferring translational energy into rotational energy. Or 2. During impacts of large particles, outer parts may roll "downhill" in the inertial field of deceleration which acts like a gravitational field, of course missing in our case. Again, friction would convert translation into rotation. A fragment may further lose even smaller parts while rolling along the target surface until it comes to rest. Fig. 2a-6 shows such a case.

Normally, large impacts result in a central pile. In the present data set, however, we discovered two examples where the center shows a shallow depression: Jessica Fig. 2b-7, and Kamil Fig. 2b-8. A stronger central core covered with more fluffy material would produce such a pattern. Upon impact, the fluffy material is shed off, producing a ring pattern of fragments, while the central particle rebounds off the target. The argument is speculative since we have no hints that our particles contain larger compact elements. However we cannot at present exclude such a possibility, in view of the STARDUST findings (Brownlee et al., 2012) and the investigations of IDPs (Matrajt et al., 2012). The central core could also be a piece of ice, which then sublimates after impact, as the target is at approximately room temperature. This would mean mineral covered ices instead of the conventional 


\section{ACCEPTED MANUSCRIPT}

concept of ice covered minerals, e.g. Greenberg and Hage, 1990. In this case, it would have consequences for our understanding of how ices and dust co-exist inside of the comet nucleus.

Linear chains of debris become sometimes apparent. An example is Adeline in Fig. 2b-9. It is an indication for high porosity and low coordination number, i.e. each element is in contact with only few neighbors.

\subsection{Summary of our observations}

Images of selected examples show a diversity of phenomena. However, each of the examples stands for a group of particles.

1. Lambert is an example of a group of particles that have a compact, undamaged appearance. Its well defined shadow shows that the contact area with the target is small. 17 such particles larger than $80 \mu \mathrm{m}$ have been found.

2: Pecine is an example for particles smaller than 50 ? $\mathrm{m}$, and which did not fragment. Fragments around a bigger impact cluster are of this size, but also very many single particles, randomly distributed across the entire target. There are more than 7000 particles $<50$ ? $\mathrm{m}$ within the present data set.

3, 4: Stefanie and Pertti are examples of particles of medium size that break up into a few fragments. 90 examples with sizes $<100 ? \mathrm{~m}$ have been detected within the present data set. In addition there are 9 examples of simple breakup at sizes $>100$ ? $\mathrm{m}$.

5: Nilda is a typical example for a catastrophic disruption with ejection of fragments to larger distances $l_{R}$ from the impact location, most probably achieved by rolling. Within the present data set 12 particles of this type have been detected having sizes between 100 and 300 ? $\mathrm{m}$.

6: Clarence is a single, special case to show rolling of individual particles and a possible loss of smaller fragments.

7: Jessica is an example of a big particle which ejects fragments into the surroundings but stays dense at its central part, thus suggesting a higher strength. Jessica also shows a central depression.

8: Kamil shows damage, yet no ejection of fragments. It also shows a central depression, and as a special feature, a trail of fragments into a preferred direction. Kamil was possibly rolling with loss of fragments before it came to rest.

9: Adeline shows some inner shattering and reveals chain like structures for its elements.

10: Jean-Baptiste shows both, a dense compact central part, and also ejection of fragments into the neighbourhood.

The particles 7-10 have in common, that they all show some damage, with loss of fragments, yet not complete disruption, although they are quite large. This points to a higher inner strength, and some outer parts having less strength. This means, strength is not always a quantity constant throughout the particle. This is not surprising, since also chemical compositions can vary across a larger particle see e.g. Paquette 2016. The present data set contains 19 examples of such mixed types with sizes larger than $100 ? \mathrm{~m}$. 


\section{ACCEPTED MANUSCRIPT}

Our observation is: Particles having an equivalent sphere diameter of less than $\sim 40$ ? $\mathrm{m}$ did not fragment. Despite of the resolution limitations we assume that there are no impact caused damages because smaller particles show higher strength. Between 50 and $100 ? \mathrm{~m}$ there is mostly breakup into a few fragments only, and above 120 ?m we observe a variety of breakups. Disruption fragments, as well as individual elements within larger, unfragmented particles, are essentially of the same size and show a very narrow size distribution of $\sim 10$ to 40 ? $\mathrm{m}$. This suggests that the impact fragmentation process can be viewed as a "falling apart" of the agglomerate's elements. Fragments are thought to exist pre-formed as elements within the dust agglomerate, and their size is not determined by fracture mechanics during the impact. Table 1 summarizes our observations for the 10 selected example particles.

\begin{tabular}{|l|c|c|c|c|c|c|c|c|}
\hline$\#$ & $\begin{array}{c}\text { Example } \\
(\mathrm{x}, \mathrm{y})[\mu \mathrm{m}]\end{array}$ & $\begin{array}{c}a \\
{\left[10^{3}\right.}\end{array}$ & $\begin{array}{c}h \\
{[\mu \mathrm{m}]}\end{array}$ & $\begin{array}{c}d \\
{[\mu \mathrm{m}]}\end{array}$ & $\begin{array}{c}d_{c_{1}} \\
{[\mu m]}\end{array}$ & $\begin{array}{c}d_{c_{2}} \\
{[\mu m]}\end{array}$ & $b r$ & type \\
\hline
\end{tabular}




\begin{tabular}{|c|c|c|c|c|c|c|c|c|}
\hline & \multicolumn{8}{|c|}{ ACCEPTED MANUSCRIPT } \\
\hline & & $\left.\mu m^{2}\right]$ & & & & & & \\
\hline 1 & $\begin{array}{l}\text { 2CF Lambert } \\
\text { Kolima.3 } \\
(8504,3804)\end{array}$ & 11.6 & 115 & 108 & - & - & no & $\mathrm{C}$ \\
\hline 2 & $\begin{array}{l}\text { 1CF Pecine Ala- } \\
\text { Kitka }(3114,5669)\end{array}$ & 1.57 & 45 & 41 & - & - & no & - \\
\hline 3 & $\begin{array}{l}\text { 2D0 Stefanie } \\
\text { Saimaa }(5648,2703)\end{array}$ & 15.2 & 45 & 87 & 15 & 40 & $\begin{array}{l}\text { s. } \\
l_{d} \approx 600 \mu m\end{array}$ & $\mathrm{R}$ \\
\hline 4 & $\begin{array}{l}\text { 2CF Pertti } \\
\text { Kolima.3 } \\
(4162,8158)\end{array}$ & 18.8 & 40 & 89 & 20 & 35 & $\begin{array}{l}\text { s. } \\
l_{d} \approx 200 \mu m\end{array}$ & $\mathrm{~S}$ \\
\hline 5 & $\begin{array}{l}\text { 2CF Nilda } \\
\text { Kolima.3 } \\
(7700,3760)\end{array}$ & 220 & 140 & 309 & 10 & 35 & $l_{R} \approx 200 \mu m$ & $\mathrm{R}$ \\
\hline 6 & $\begin{array}{l}\text { 2CF Clarence } \\
\text { Kolima.3 } \\
(4428,9414)\end{array}$ & 3.7 & 68 & 63 & 15 & & d. & - \\
\hline 7 & $\begin{array}{l}\text { 2CF Jessica } \\
\text { Lummene. } 2 \\
(1277,4293)\end{array}$ & 232 & 40 & 207 & 15 & 40 & $\begin{array}{l}\text { d. } \\
150 \mu m\end{array} l_{R} \approx$ & $\mathrm{S}$ \\
\hline 8 & $\begin{array}{l}\text { 3D0 Kamil } \\
\text { Ukonvesi } \\
(7452,9230)\end{array}$ & 120 & 50 & 179 & 15 & 30 & d. & $\mathrm{S}$ \\
\hline 9 & $\begin{array}{l}\text { 2CF Adeline } \\
\text { Kolima.3 } \\
(5518,3926)\end{array}$ & 35.8 & 50 & 120 & 20 & 20 & d. & $\mathrm{G}$ \\
\hline 10 & $\begin{array}{l}\text { 2CF Jean-Baptiste } \\
\text { Kolima.3 } \\
(7120,3190)\end{array}$ & 103 & 100 & 214 & 15 & 40 & d. & GS \\
\hline
\end{tabular}

Table 1: Summary of observations for the 10 example particles of Fig. $2 a$ and $2 b$.

br: breakup behavior. s.: simple breakup into few pieces, cat: catastrophic breakup,

d: impact damage. $h$ : height, as derived from the shadow; $d$ : equivalent sphere diameter of the incoming dust: $d=\left(\frac{6}{\pi} \cdot a \cdot h \cdot \varepsilon\right)^{1 / 3}, \varepsilon=1 / 2$ assumed throughout. $d_{c_{1}}, d_{c_{2}}:$ lower and upper fragment (or element) diameters. $l_{R}$ : characteristic rolling length; $l_{d}$ : mean next nearest neighbour distance between background particles.

type: Categories of Langevin et al, 2016: $\mathrm{C}=$ "compact", $\mathrm{S}=$ "shattered", $\mathrm{R}=$ "rubble pile", $\mathrm{G}=$ "glued cluster", GS= mixed: "glued cluster shattered”, “_-“: particle not part of Langevin et al. data set.

( $\mathrm{x}, \mathrm{y}$ : positions relative to lower left corner of the $1 \mathrm{x} 1 \mathrm{~cm}^{2}$ target plate).

\section{An estimate of the mechanical strength from breakup conditions}


The strength of cometary matter is mostly investigated from the viewpoint of comet formation (e.g. Blum et al., 2008, Güttler et al. 2009, Blum et al., 2014). Here, we focus on the state of the material originating from the nucleus of the comet. It may have been processed during the comet's passages by the sun, and also from its ejection until the arrival at our instrument. Having lost its volatiles by sublimation (Clark et al., 2004), it finally became the fluffy material we see in our collections (Schulz et al., 2015).

We estimate the strength of the incoming dust from properties of the apparent breakup. We cannot, however, make use of the classical continuum theories (Thomson 1973) since we have no data on material properties like Young modulus, surface tension etc. There are, however, methods more adequate for low density granular materials starting from building blocks of sub-micrometer scales (Blum et al. 2014). Breakup is then simulated numerically by detailed modeling of grain interactions, e.g. rolling (Kimura et al. 2015). The results are compared with laboratory experiments with fine dust of known material properties. The applicability of such concepts to "real" cometary dust depends on the physical and chemical nature of the smallest sub-micrometer building blocks. These probably consist of a mixture of minerals, embedded in a non-volatile organic material (Kissel and Krueger 1987, Matrajt et al. 2012, Bradley 2012, Engrand et al. 2015), holding them together to form compact grains. Little is known, however, of their mechanical properties. We therefore use for our data interpretation simplified models only to derive bounds for the strength of the dust agglomerate before its impact. Particle disruption during impact depends on a combination of both, tensile, and shear forces. Because our data do not allow to distinguish between the tensile and the shear strengths, we use the general term "strength". However, recent experimental and numerical investigations of agglomerate systems indicate that the difference between shear- and tensile strength is small for porous dust with filling factors between 0.3 and 0.6 (Güttler et al. 2009, Seizinger et al., 2012, 2013). Also Kirchner et al. (2002) measured both tensile and shear forces for low density dry snow at $\rho \approx 0.17 \mathrm{~g} / \mathrm{cm}^{3}$ and found no difference. Our case is an example for the process-dependent definition of strength, as described by e.g. Housen and Holsapple, 1990. According to our data, impacts at a few $\mathrm{m} / \mathrm{s}$ are sufficient to cause disintegration into fragments of several $10 \mu \mathrm{m}$, but at much higher impact speeds the dust may break them up into even smaller pieces.

\subsection{Constraints for the strength from the energy density}

One aspect is to use the impact induced energy density (mechanical energy per unit volume). If it exceeds the strength of the particle, breakup occurs. Similar simplified reasoning has been applied to treat e.g. the breakup of a large body by a hypervelocity impact of a smaller projectile c.f. Housen and Holsapple, 1990., the breakup of particles during atmospheric entry (Ceplecha and McCrosky, 1976), or the breakup of Shoemaker-Levy 9 by gravitational forces during the 1992 Jupiter encounter (Scotti and Melosh 1993). In our case the incoming dust particle dissipates its whole kinetic energy during the impact, therefore the energy density is:

$\sigma_{e d}=\frac{\rho}{2} \cdot v^{2}$

where $\rho$ is the dust particle's mean mass density and $v$ is the impact speed. In terms of continuum mechanics this would correspond to the pressure increase at the stagnation point. For atmospheric entry the expression is similar, $\rho$ being the air density at the respective altitudes and $v$ is the entry speed. Several km/s are required for meteor breakup. In our case, $\rho$ is the orders of magnitude higher solid state density and this is the physical cause that speeds of some $\mathrm{m} / \mathrm{s}$ are sufficient for the breakup. If a particle fragments upon impact, then its strength has to be smaller than the value given by Eq.(1). In this case Eq.(1) represents an upper bound for the strength, whereas for a non-fragmenting particle it represents a lower bound. Dust particle speeds for the time of observation (Aug. 2014 to Feb. 2015) have been measured by the GIADA instrument onboard ROSETTA and we take the values reported by Rotundi et al., 2015, Della Corte et al., 2015 and assume that the dust particles we observe have similar speeds. Their velocity distribution shows a mean value of $3.5 \mathrm{~m} / \mathrm{s} \pm 1.5 \mathrm{~m} / \mathrm{s}$. For the size dependent mass density we assume a power law: 
$\rho=\rho_{0} \cdot\left(r / r_{0}\right)^{-b}$

To derive the constants we start from the smallest units of $\sim 0.1 \mu \mathrm{m}$ in radius, and assume them to be compact. We further assume that the volatiles have sublimated (with possible exceptions as discussed in Sec. 2.2.3), i.e. only minerals and refractory carbon-rich organics are left whose mean density we estimate to about $2.5 \mathrm{~g} / \mathrm{cm}^{3}$, based on the composition estimates of Greenberg and $\mathrm{Li}$ (1999). This value is in approximate agreement with the evaluation by Rotundi et al. (2015) of the density of compact dust measured around the nucleus of C67P $\left(1.9 \pm 1.1 \mathrm{~g} / \mathrm{cm}^{3}\right)$. Further information can be derived from our images of not-fragmented dust agglomerates, see e.g. the particles of Fig. $2 b-7,8$, and 9 . The packing of their elements looks being far from extremely porous (volume filling factors as low as $\approx 0.15$, see cf. Blum and Schräpler 2006) but also less than randomly close packed (fill factor $\approx 0.6$ ). So we take an intermediate value for the filling factor of $\sim 0.4$, which, in turn leads to a mass density decrease by a factor of $1 / 0.4=2.5$ per size decade. This is supported by the data, as the individual elements in the images have sizes of roughly $1 / 10$ of the parent particle $(b \approx \log (2.5))$. Assuming, that such an intermediate packing density also prevails in the submicron domain, results in $\rho=\rho_{0} \approx 1 \mathrm{~g} / \mathrm{cm}^{3}$ at $r=r_{0} \approx 1 \mu \mathrm{m}$, consistent with densities determined in the coma of Halley (Maas et al., 1990, Hornung and Kissel 1994). Dust of $\sim 100 \mu \mathrm{m}$ in radius would then have a density of $0.16 \mathrm{~g} / \mathrm{cm}^{3}$. This, however, is already less than the comet's mean density of $0.533 \mathrm{~g} /$ $\mathrm{cm}^{3}$ (Pätzold et al. 2016), which means that we have to conclude, that the dust we collected, besides having lost its volatiles, has been further processed at the surface of the nucleus, and/or on its way to the instrument (Schulz et al, 2015). We did use Eq.(2) for an extrapolation up to a few $100 \mu \mathrm{m}$. Further extrapolation should only be done with extra care! A power law dependence of the mass density on size is also known from Ballistic Cluster-Cluster Aggregation analysis cf. e.g. Mukai et al., (1992).

Fig. 3 shows how the energy density $\sigma_{e d}$ depends on the velocity for various values of the mass density $\rho$. Sizes in this plot are in radius $r=d / 2$. 


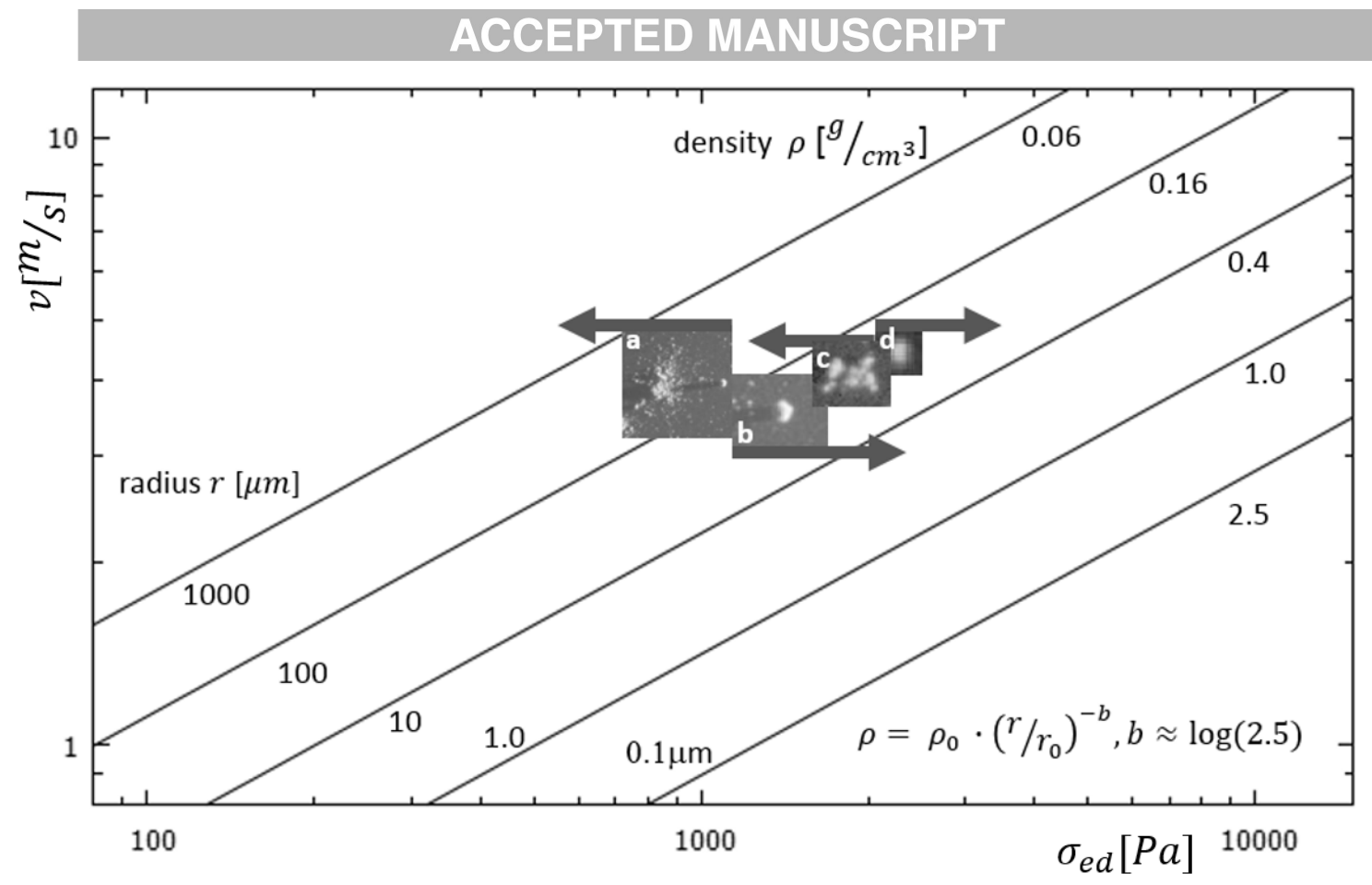

Fig. 3: Energy density $\sigma_{e d}$ in dependence on impact speed $v$ for various mass densities $\rho$ of the impacting dust particle together with the possible location of some particles of Fig. 2.

a: catastrophic breakup, Nilda,

b: big compact particle, Lambert,

c: simple breakup into few pieces, Pertti,

d: small compact particle, Pecine.

Left pointing arrows: Position in diagram is an upper bound for strength; right pointing arrows:

Position in diagram is a lower bound for strength.

The energy density argument used above, leads to the following approximate constraints for particle strength:

i) Catastrophic breakup, e.g. Nilda. Its position (a) is indicated for the estimated speed and mass densities from Eq. (2). For fragmenting particles such as Nilda, the energy density value gives an upper bound for the strength, which for Nilda is $\sim 1000 \mathrm{~Pa}$.

ii) The simple breakup events show similarities with the catastrophic breakup events. The main difference is, that the incoming dust has a smaller size (by a factor of 4 for the examples of Fig. 2a), and hence a higher mass density (Eq. 2). Breakup results in only a few fragments. The resulting position of 2CF Pertti in Fig. 3 (c) indicates that the upper bound for its strength should be slightly higher than $1000 \mathrm{~Pa}$.

iii) For the case of no breakup, strength has to be higher than the energy density value. Fig. 3 shows the corresponding position of $1 \mathrm{CF}$ Pecine (d) at about $2000 \mathrm{~Pa}$. The radius of Pecine (about $20 \mu \mathrm{m}$ ) is already down to the size of typical elements within larger dust particles (and also of their fragments upon impact) such that its integrity appears logical. Much higher impact speeds would be needed to further break up these small particles. The position of non-fragmenting large particle $2 \mathrm{CF}$ Lambert (b) is slightly above $1000 \mathrm{~Pa}$.

iv) When there is "shedding from a stronger central core", this indicates that the particle might not be homogeneous in strength (e.g. Jean-Baptiste, Fig. 2b-10 or Jessica Fig. 2b-7). From the size of the fragments in its neighbourhood, one would assume a loose outer part with a strength comparable to 


\section{ACCEPTED MANUSCRIPT}

the case of fragmenting dust, and an inner core comparable to the case of compact dust, "glued cluster" in Langevin et al. (2016).

The energy density concept also leaves a possibility to compare to experiments dealing with other ranges for the parameters. For example Setoh et al. (2007) report on collisional disruption of weakly sintered porous targets at impact speeds 10 times higher than ours, and particle densities 10 times higher, resulting in strength values 3 orders of magnitude higher, however, consistent with the our simple energy density model. Beitz et al. (2011) performed impact experiments with cm-sized agglomerates consisting of spherical monodisperse $\mathrm{SiO}_{2}$ grains and found catastrophic disruption from $2 \mathrm{~m} / \mathrm{s}$ onward corresponding to an energy density of $\approx 1600 \mathrm{~Pa}$ for a volume filling factor of $\approx$ 0.4 , close to the tensile strengths measured by Blum et al. (2006) for such agglomerates.

Recently, Fulle et al. (2015) discussed the possibility of extremely low densities of about 1 $\mathrm{kg} / \mathrm{m}^{3}$ (like the aerogel in the Stardust capture cells). For these particle detections, very low speeds, down to $0.1 \mathrm{~m} / \mathrm{s}$, were observed by GIADA, however, these particles may be seen by GIADA only which has a wider field of view than COSIMA. The low particle speeds are explained by particle deceleration by electrostatic forces between charged particles and the Rosetta spacecraft surface potentials. COSIMA might not collect such dust particles on its targets since the targets are located well within the spacecraft behind the $0.15 \mathrm{~m}$ long entry funnel with a field-of-view of $15^{\circ} \times 23^{\circ}$. In our present images, and with our optical resolution, we do not see such big changes in morphology that would imply mass density changes of 2 to 3 orders of magnitude. According to Langevin et al. 2016, we observe a steady transition from compact dust ("breccia") to "clusters" which in some cases look more rigid ("glued clusters") or show signs of beginning breakup ("shattered cluster") or finally the ones which break up catastrophically ("rubble piles"). We shall, however, keep an eye on the issue in the future until end-of-mission.

The estimated strength values hold for speeds of a few $\mathrm{m} / \mathrm{s}$. The resulting strength bounds change, should variations in $v$ be larger than assumed. For example 'no-fragmentation' can be explained by high strength, but also by low impact speeds. We'll give values for the strength vs. energy density in Sec. 3.4 below. 


\section{ACCEPTED MANUSCRIPT}

3.2 Information on the binding forces from the deceleration forces.

It is possible to calculate the binding forces between the agglomerate's elements by estimating the inertial forces due to deceleration. To this respect COSIMA can be regarded as an "impact experiment in space" (Fig. 4).
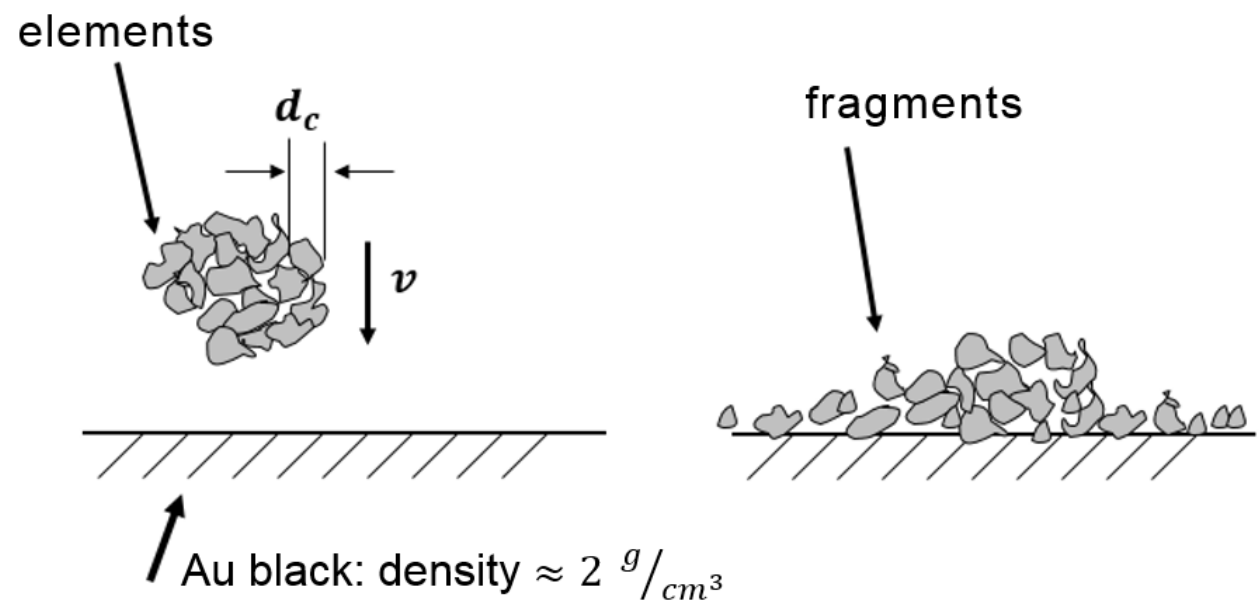

\section{Fig. 4: An impact fragmentation experiment in space.}

Take one of the constituent elements "c" at the periphery of the dust agglomerate. It is bound to the agglomerate and for the moment we assume that the binding force is proportional to the element's equivalent sphere diameter:

$F_{b i n d}=C \cdot d_{c}$

To break it off the agglomerate during impact, the force caused by the deceleration from speed $v$ to zero, within the stopping length $s$, has to be larger than the binding force:

$F_{d e c}=m_{c} \cdot \frac{v^{2}}{2 s}=\frac{\pi d_{c}{ }^{3}}{6} \cdot \rho_{c} \cdot \frac{v^{2}}{2 s}>F_{b i n d}$.

The typical stopping length $s$ is known from the surface structure of the collection substrate (porous gold, see Hornung et al., 2014) which has a typical roughness in the order of $10 \mu \mathrm{m}$, see Fig. 5. We take this value as an upper limit for $s$. We also take the size dependence of the mass density from Eq.(2) which should also hold for the density $\rho_{c}$ of the individual elements.

For the force constant $C$ in Eq. (3) we start with an estimate, derived from van der Waals interaction of spherical elements: $C=\frac{A}{24 \cdot D^{2}}, D \approx 0.4 \mathrm{~nm}$ with the Hamaker constant of dry minerals under vacuum conditions: $A \approx 10^{-19} \mathrm{~J}$, leading to $C \approx 2.6 \cdot 10^{-2} \mathrm{~N} / \mathrm{m}$ (cf. Israelachvili, 2011).

The results for the deceleration- and binding forces are shown in Fig. 6 for various impact speeds around $3.5 \mathrm{~m} / \mathrm{s}$. The crossover between binding and deceleration forces then defines the lower size 


\section{ACCEPTED MANUSCRIPT}

limit for fragments, which can be detached from a dust agglomerate $\left(d_{c}\right.$ between 10 and $\left.15 \mu \mathrm{m}\right)$. From our optical inspection we have no indication for much smaller fragments. However, since 10-15 $\mu \mathrm{m}$ is at the limit of our optical resolution of $10 \mu \mathrm{m}$, we look for information from other sources. Some indication comes from our SIMS (secondary ion mass spectrometry) measurements, Hilchenbach et al. (2016): If smaller fragments were present, then they should contribute significantly to the SIMS signal in the spaces between the dust fragments, since SIMS is sensitive to surfaces only. We see, however, a clear change of the ion signal from "on the dust" to "off the dust" down to values, typical for an empty target. More information should eventually come from the MIDAS team (an atomic force microscope on board), once their data evaluation is completed. From the agreement of the observed minimum fragment sizes with the values shown in Fig. 6 we conclude that the assumed force constant is reasonable and we will use it for our further modelling below. It represents a lower limit for the inter-element force not only because it reproduces the observed lower cutoff for the fragment size but also because we have used an upper limit for the stopping length. In the above estimate we did not distinguish between tearing and shear forces, which is suggested by the above discussed approximate coincidence of tensile- and shear strengths for porous agglomerates.

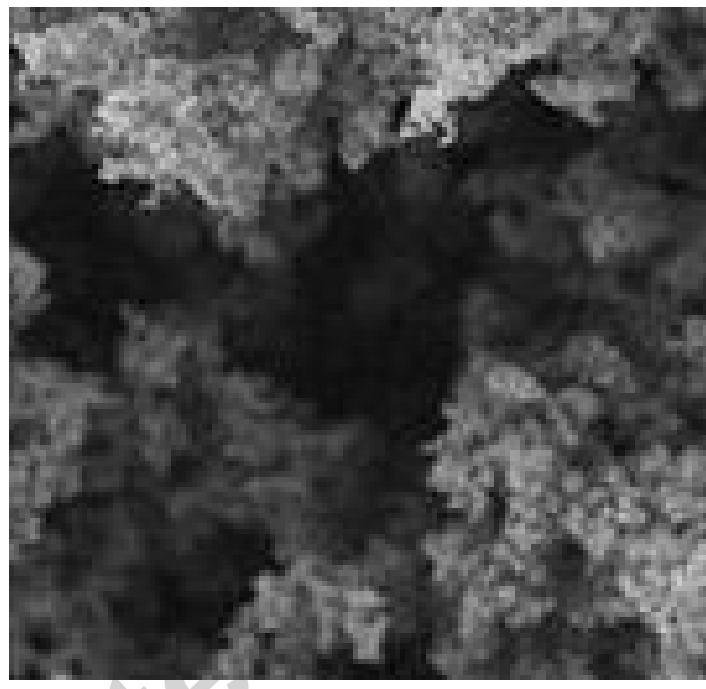

Fig. 5: Surface structure of Au black (SEM image: $8 \times 8 \mu m^{2}$ ). Note that this image is smaller than a single COSISCOPE pixel $\left(14 \times 14 \mu m^{2}\right)$. At COSISCOPE image resolution the Au black appears locally smooth. 


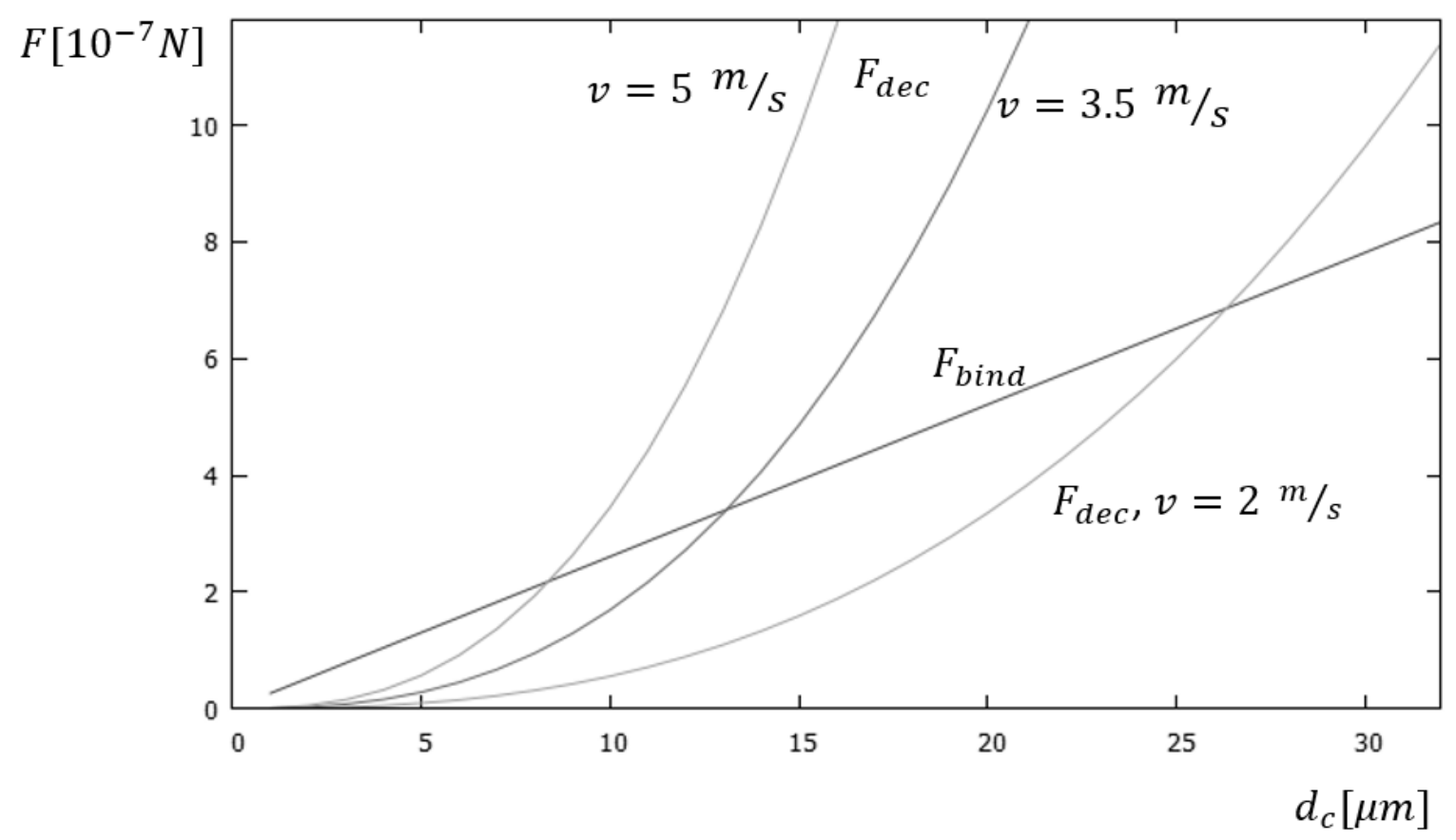

Fig. 6: Size dependence of the forces: The crossover point defines the minimum size of elements which can be detached by the impact from the parent dust particle (examples $v \approx 2,3.5$, $5 \mathrm{~m} / \mathrm{s})$.

The knowledge of forces can be used to describe the rolling of fragments along the target, away from the impact site as mentioned in Sec. 2.2.2. Since there is no gravity, they can be kept at the gold-black surface only by adhesion. According to Israelachvili, (2011) this force is about twice as large as the inter-element force: $F=2 \cdot C \cdot d_{c}$. The rolling resistance is generally less than the force in normal direction to the surface by some factor, known as "rolling resistance coefficient" $c_{R}$, a quantity which in technical systems assumes values between 0.01 and 0.1 (Bower, 2010). Assuming the total impact energy being dissipated in rolling we get an upper bound for the rolling length: $l_{R}=\frac{\frac{m_{C}}{2} \cdot v^{2}}{F \cdot c_{R}}$. Using the fragment's mass density from Eq.(2) we get for the example of $v=3.5 \frac{\mathrm{m}}{\mathrm{s}}$ and $d_{c}=20 \mu \mathrm{m}: l_{R}=$ $100 \ldots 1000 \mu \mathrm{m}$. This agrees reasonably well with observed movements of fragments away from the impact site in the order of a few $100 \mu \mathrm{m}$ (see Fig. 2c and Table 1). So movement by rolling is plausible. 


\section{ACCEPTED MANUSCRIPT}

\subsection{Estimating the strength of the incoming dust from the size distribution of its fragments.}

We try here to derive the strength of the incoming dust agglomerate for the case it fragments upon impact. As sizes of its elements we take the sizes of the fragments we observed (see Sec. 2.3). If we would split such an agglomerate into two parts along an imaginary dividing surface the strength would then be defined as the force necessary to tear them apart divided by the area of the surface:

$\sigma=F_{\text {bind }} \cdot n_{c} \cdot d_{c}, n_{c}=\frac{\rho}{m_{c}}$

where $n_{c} \cdot d_{c}$ is the number of elements per unit area at the dividing surface and $\rho$ is the mean density of the agglomerate. The observed chain structures within not-fragmented dust supports such a simple way of reasoning which involves breakup of binary bonds. Inserting van der Waals interaction from Eq. (3), and assuming, as a first step, that the elements have equal size results in:

$\sigma=\frac{3}{\pi} \cdot C \cdot \frac{\rho}{\rho_{c}} \cdot \frac{1}{r_{c}}=\frac{3}{\pi} \cdot C \cdot \emptyset \cdot \frac{1}{r_{c}} \quad$,

where we have switched to radius $r_{c}=d_{c} / 2$, and $\emptyset=\rho / \rho_{c}$ is the volume filling factor, i.e. the fraction of the total volume occupied by the elements. Eq. (6) reflects that strength derived from van der Waals adhesion forces is inversely proportional to the size of the elements, as known from powder technology (e.g. Rumpf, 1958). However, since we have observed a range of sizes we have to extent the formalism accordingly. The van der Waals adhesion force between a pair of spheres of radii $r_{c}, r_{c}^{\prime}$ is: $F_{b i n d}=2 C \cdot\left(2 r_{c} \dot{r}_{c} / r_{c}+\dot{r}_{c}\right)$ (Israelachvili, 2011). For a distribution of sizes, $f\left(r_{c}\right)$, the sum of the contributions of the pairs of elements across the dividing surface is:

$$
\sigma=\sigma_{0} \cdot \frac{\int_{r_{c_{1}}}^{r_{c_{2}}} f(r) \cdot r \cdot\left\{\int_{r_{c_{1}}}^{r_{c_{2}}} f(\dot{r}) \cdot \frac{2 r \dot{r}}{r+\dot{r}} d \dot{r}\right\} d r}{\int_{r_{c_{1}}}^{r_{c_{2}}} f(r) \cdot \frac{\rho_{c}(r)}{\rho_{0}} \cdot r^{3} d r},
$$

where:

$\sigma_{0}=\frac{3}{\pi} \cdot C \cdot \frac{\rho}{\rho_{0}} \cdot \frac{1}{r_{0}} ; \rho_{0}=1000 \frac{\mathrm{kg}}{\mathrm{m}^{3}} ; r_{0}=10^{-6} \mathrm{~m}$.

The size dependence of the element densities $\rho_{c}(r)$ is taken from Eq.(2) and integration is over the observed size range of the fragments or elements : $r_{c_{1}}<r_{c}<r_{c_{2}}$ using

$f\left(r_{c}\right)=a \cdot r_{c}{ }^{-c}, \quad$,

where the constant $c$ is taken from the data of Sec. 2.2.2. Normalization to one within the observed size range gives $a=(1-c) /\left(r_{c_{2}}^{1-c}-r_{c_{1}}^{1-c}\right)$ and a simple analytical solution for Eq.(7):

$$
\sigma=\sigma_{0} \cdot \frac{a \cdot(4-b-c)}{(2-c)^{2}} \cdot \frac{\left(r_{c 2}^{2-c}-r_{c 1}{ }^{2-c}\right)^{2}}{\left(r_{c 2}{ }^{4-b-c}-r_{c 1}{ }^{4-b-c}\right)} \text {. }
$$


The volume filling factor for the multi-size case is given by the ratio between the incoming dust's mean density $\rho$ and the mean element density $\overline{\rho_{c}}$ averaged over the size distribution:

$\varnothing=\rho / \overline{\rho_{c}} \quad, \overline{\rho_{c}}=\frac{\int_{r_{c 1}}^{r_{c 2}} f(r) \cdot \rho_{c}(r) \cdot r^{3} d r}{\int_{r_{c 1}}^{r_{c 2}} f(r) \cdot r^{3} d r}=\left(\frac{4-c}{4-c-b}\right) \cdot\left(\frac{r_{c 2}{ }^{4-c-b}-r_{c_{1}}{ }^{4-c-b}}{r_{c_{2}}{ }^{4-c}-r_{c_{1}}{ }^{4-c}}\right)$.

In order to demonstrate the properties of the formalism, we give an example for a typical particle size for fragmenting particles: radius $100 \mu \mathrm{m}$, mean density $\rho=0.16 \mathrm{~g} / \mathrm{cm}^{3}$. Fig. 7 shows the result of Eq.(7) for this case and Table 2 contains resulting strengths for various combinations of minimum and maximum fragment sizes $r_{c_{1}}, r_{c_{2}}$. The values are in the order of $10^{3} \mathrm{~Pa}$. More specific application to the individual particles of our data set (Sec. 2) is performed in Sec. 3.4 below.

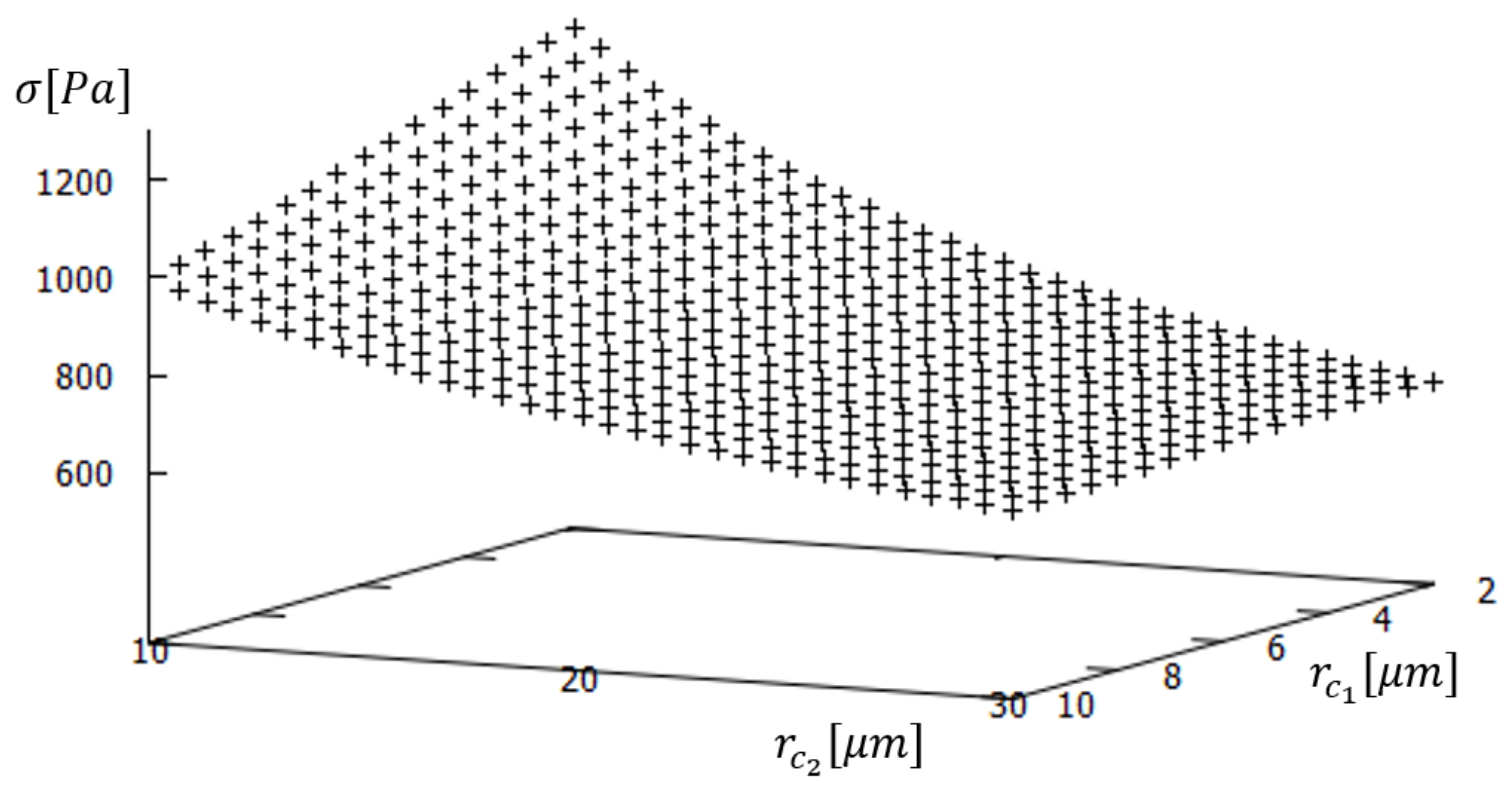

Fig. 7: Mechanical strength [Pa] in dependence on fragment sizes for the example of $\rho=0.16 \mathrm{~g} / \mathrm{cm}^{3} \sigma_{0}=3979 \mathrm{~Pa}$. Every point on the plotted surface corresponds to a window of lower and upper sizes.

\begin{tabular}{|l|l|l|l|}
\hline \multicolumn{1}{|c|}{$r_{c_{1}}[\mu m]$} & $r_{c_{2}}[\mu m]$ & $\varnothing$ & $\sigma[\mathrm{Pa}]$ \\
\hline 5 & 20 & 0.42 & 830 \\
\hline 5 & 10 & 0.35 & 1160 \\
\hline
\end{tabular}




\begin{tabular}{|l|l|l|l|}
\hline 1 & 20 & 0.36 & 850 \\
\hline 1 & 10 & 0.29 & 1300 \\
\hline 1 & 5 & 0.23 & 1940 \\
\hline
\end{tabular}

Table 2: Example values of Eq. (7) for various size bounds as well as resulting strength values for a mean density of $0.16^{\mathrm{g}} / \mathrm{cm}^{3}$ corresponding to a $\mathrm{r}=100 \mu \mathrm{m}$ sized incoming dust particle.

To estimate the uncertainties of the method it is helpful to know how sensible the input parameters enter into Eq.(7). Table 3 contains the corresponding sensitivity factors for deviations from a reference set of input parameters. It shows that the largest fragment $r_{c_{2}}$ influences the strength more than the smallest one $r_{c_{1}}$ for the example chosen which is typical for a large fragmenting particle. Hence uncertainties of $r_{c_{1}}$ caused by resolution limitations do not enter significantly. Also the steepness $c$ of the size distribution enters with low sensitivity, which justifies choosing an approximate value of 3 (see Sec. 2.2.2). The force constant $C$ enters linearly $\left(s_{1}=1\right)$. The value chosen is for mineral materials and may be lower by a factor of two for refractory organics (Israelachvili 2011). However our data analysis on forces (Sec. 3.2) suggests values close to the mineral value. The density exponent $b$ enters almost linearly. When considering the force constant $C$ as a lower bound for the element interaction, the main remaining uncertainty results from the density. It is expected to be accurate within a factor of two at high values of porosity $(1-\emptyset)$ leading to a total uncertainty for the strength in the same order.

\begin{tabular}{|c|c|c|}
\hline $\begin{array}{c}\text { parameter } p_{i} \\
\begin{array}{c}\text { Force constant } C \\
{[N / m]}\end{array}\end{array}$ & $p_{i}^{\text {ref }}=\left|\frac{p_{i}}{\sigma} \cdot \frac{\Delta \sigma}{\Delta p_{i}}\right|$ \\
\hline$r_{c_{1}}[\mu m]$ & 0.026 & 1.0 \\
\hline$r_{c_{2}}[\mu m]$ & 5 & 0.07 \\
\hline$c$ & 20 & 0.5 \\
\hline$b$ & 3 & 0.4 \\
\hline$b$ & 0.4 & 0.9 \\
\hline
\end{tabular}

Table 3: Sensitivity factors $s_{i}$ for a $\pm 1 \%$ variation of the value of input parameters $p_{i}$ around a reference state $p_{i}^{\text {ref }}\left(\sigma_{\text {ref }}=830 \mathrm{~Pa}\right)$.

Within the simplicity of the approach, Eq. (7) provides a rough quantification of the strength that holds the elements together as an agglomerate. Defined as the force per unit area necessary to separate the elements from their attaching neighbors it represents an estimation of the tensile strength. For the present examples the area density of elements at the dividing surface is very low which is also in favor of a simple description. For example Nilda and Pertti (Fig. 2a) have about 100 and 10 elements at the cross section dividing them into two halfs, respectively. Due to the small total number of elements within the 
agglomerate (up to $\sim 1500$ for the largest particles), known effects associated with crack and flaws appearing at weakest zones caused by an inhomogeneous distribution of stronger and weaker areas (Reynolds et al., 2005) are dismissed in our case. The narrowness of the element's size distribution prevents the accuracy of this simple scheme from being compromised by the situation of many smaller dust particles surrounding a much larger one (Sanchez and Scheeres 2014). Moreover, it is known that when the coordination number is high, not all the contacts are transmitting the stress with the full adhesion force, and, when the coordination number is low, the agglomerate tends to stretch in the direction of the stress, forming chains (Seizinger et al. 2013, Blum et al., 2006). In view of the many variables at play, not only involving the packing perspective, but for example also the premise of perfect sphere contact, we prefer to ensure easiness in the formalism within a first data assessment.

\subsection{Example dust particles}

We summarize in Table 4 the strength estimates for the example the particles of Sec. 2. The values refer to the intact dust particle before its impact onto the target. Both methods are included: $\sigma_{f s}$ using the fragment sizes as in Sec. 3.3, and $\sigma_{e d}$ from energy density as in Sec. 3.2. $\sigma_{e d}^{(1)}$ is calculated for a mean impact speed of $3.5 \mathrm{~m} / \mathrm{s}$. However, the assumption of a constant impact speed for all particle sizes is likely only if all particles up to a few $100 \mu \mathrm{m}$ in size are lifted together from the comet's surface and break up to smaller sizes afterwards. Should the differentiation already occur during the liftoff-process then there may be a bigger spread in speed. Models assume $v \propto d^{-0.5}$ Agarwal et al. (2007). To estimate the influence on the strength, Table 4 additionally contains energy density values $\sigma_{e d}^{(2)}$ for the variable speed case. We assume that both approaches should give the same result for the largest dust particle observed by us: Nilda. Due to the arbitrariness of this way of fitting, the values for $\sigma_{e d}^{(2)}$ in Table 4 are put in brackets.

1: For the non-fragmenting particle Lambert the energy density values are a lower limit.

2: The same holds for the small Pecine particle.

3: and 4: The Stefanie and Pertti particles are fragmenting, so an upper bound from energy density as well as fragment size values are available.

5: For the catastrophically disrupting particle Nilda energy density and fragment size values nearly coincide. As the energy density value is used to fit a size-dependent speed, both values of the upper bound from energy density are equal.

6 to 10: Since these particles do not show clear fragmentation, the value derived from the sizes of the elements might not be relevant. At most, it then represents a hypothetical lower limit if we assume that they are bound by van der Waals forces only. Their morphology, however, suggests, that an additional inter-element glue is present (Langevin et al. 2016), which provides them with more strength. These values are therefore in parentheses. The values of the energy density, however, are relevant, since there is indeed some damage. They represent an upper bound for the value of strength. The fact that the lower energy density value "ed" of No. 6 (Clarence) slightly exceeds the element size value "fs" is of no relevance in view of the large uncertainties within both models.

The values of the mass density listed, are within a range which have been derived for the coma of comet Halley (Maas et al. 1990). The volume filling factors reach values of random close packing $\approx 0.6$ for particles consisting of few elements, whereas for larger dust particles values seem to be 


\section{ACCEPTED MANUSCRIPT}

0.4 . A note of caution: these values hold for the packing of the "elements". Because they themselves have an inner porosity, the total resulting porosity may well be above $90 \%$ (volume filling less than 0.1).

\begin{tabular}{|c|l|l|l|l|l|l|l|l|l|l|}
\hline$\#$ & \multicolumn{1}{|c|}{ Example } & $b r$ & $\begin{array}{c}d \\
{[\mu \mathrm{m}]}\end{array}$ & $\begin{array}{c}d_{c_{1}} \\
{[\mu \mathrm{m}]}\end{array}$ & $\begin{array}{c}d_{c_{2}} \\
{[\mu \mathrm{m}]}\end{array}$ & $\varnothing$ & $\begin{array}{c}\rho \\
\mathrm{kg}\end{array}$ & $\begin{array}{c}\sigma_{f s} \\
{[\mathrm{~Pa}]}\end{array}$ & $\begin{array}{c}\sigma_{\text {ed }}^{(1)} \\
{[\mathrm{Pa}]}\end{array}$ & $\begin{array}{c}{\left[\sigma_{\text {ed }}^{(2)}\right]} \\
{[\mathrm{Pa}]}\end{array}$ \\
\hline 1 & $\begin{array}{l}\text { 2CF Lambert } \\
\text { Kolima.3 }\end{array}$ & no & 108 & - & - & - & 204 & - & 1250 & {$[3560]$} \\
\hline 2 & $\begin{array}{l}\text { 1CF Pecine } \\
\text { Ala-Kitka }\end{array}$ & no & 41 & - & - & - & 302 & - & 1850 & {$[14070]$} \\
\hline
\end{tabular}


ACCEPTED MANUSCRIPT

\begin{tabular}{|l|l|l|l|l|l|l|l|l|l|l|}
\hline 3 & $\begin{array}{l}\text { 2D0 Stefanie } \\
\text { Saimaa }\end{array}$ & s. & 87 & 15 & 40 & 0.63 & 223 & 1080 & 1370 & {$[4870]$} \\
\hline 4 & $\begin{array}{l}\text { 2CF Pertti } \\
\text { Kolima.3 }\end{array}$ & s. & 89 & 20 & 35 & 0.63 & 220 & 1110 & 1350 & {$[4660]$} \\
\hline 5 & $\begin{array}{l}\text { 2CF Nilda } \\
\text { Kolima.3 }\end{array}$ & cat. & 309 & 10 & 35 & 0.33 & 135 & 826 & 825 & {$[825]$} \\
\hline 6 & $\begin{array}{l}\text { 2CF Clarence } \\
\text { Kolima.3 }\end{array}$ & d. & 63 & 15 & 20 & 0.60 & 254 & $(1710)$ & 1560 & {$[7690]$} \\
\hline 7 & $\begin{array}{l}\text { 2CF Jessica } \\
\text { Lummene.2 }\end{array}$ & d. & 207 & 15 & 40 & 0.44 & 158 & $(790)$ & 967 & {$[1440]$} \\
\hline 8 & $\begin{array}{l}\text { 3D0 Kamil } \\
\text { Ukonvesi }\end{array}$ & d. & 179 & 15 & 30 & 0.43 & 167 & $(960)$ & 1020 & {$[1770]$} \\
\hline 9 & $\begin{array}{l}\text { 2CF Adeline } \\
\text { Kolima.3 }\end{array}$ & d. & 120 & 20 & 20 & 0.49 & 196 & $(1220)$ & 1200 & {$[3110]$} \\
\hline 10 & $\begin{array}{l}\text { 2CF Jean- } \\
\text { Baptiste } \\
\text { Kolima.3 }\end{array}$ & d. & 214 & 15 & 40 & 0.43 & 156 & $(780)$ & 950 & {$[1370]$} \\
\hline
\end{tabular}

Table 4: Values of the strength for dust particles before their impact onto the target as derived from the distribution of fragment sizes ("fs", see Sec. 3.3) and the energy density ("ed", see Sec. 3.1) for the 10 examples of Sec. 2. Values in parenthesis are based on element sizes of the non-fragmenting particle.

br: breakup behavior. s.: simple breakup into few pieces, cat.: catastrophic breakup,

d.: damage.

$d$ : equivalent sphere diameter : $d=\left(\frac{6}{\pi} \cdot a \cdot h \cdot \varepsilon\right)^{1 / 3}, \varepsilon=0.5$ assumed throughout.

$d_{c_{1}}, d_{c_{2}}$ : lower and upper fragment or element diameters.

$\emptyset$ : volume filling factor.

$\rho$ : intact incoming agglomerate's mean density;

$\sigma_{f s}$ : strength from analysis of the distribution of fragments or elements ( $c=3.9$ for Nilda, $c=$ 2.3 for Stefanie and Pertti, $c=3$ for the values in parentheses).

$\sigma_{e d}^{(1)}$ : strength from the energy density, assuming a mean impact speed of $v=3.5 \mathrm{~m} / \mathrm{s}$;

$\left[\sigma_{e d}^{(2)}\right]$ : strength from the energy density, and size dependent speed: $v=3.5 \cdot(d / 309)^{-0.5}$

\section{Discussion and summary}

A first analysis has been performed of the impact fragmentation behavior of dust particles, collected by the COSIMA instrument onboard ROSETTA in the collection time interval from Aug. 2014 to Feb. 2015, when Comet Churyumov-Gerasimenko was still on its way to the Sun, and ROSETTA was at 10 to $100 \mathrm{~km}$ from its nucleus. Their sizes span a range between some $10 \mu \mathrm{m}$ and $300 \mu \mathrm{m}$. Many of them broke up upon impact, revealing details about their interior. We were able to derive quantitative bounds for the mechanical strength of these dust particles for which no previous data existed. 
There is a general trend: larger dust particles fragmented while most of the smaller ones remained undamaged. The subsystem COSISCOPE provided images with a resolution of about $10 \mu \mathrm{m}$. It enabled to see the morphology of the undamaged dust as well as of fragmented dust. The fragmented and many of the undamaged dust particles show an agglomerate structure. The size of their sub-units has been found to be in the order of a few tens of microns. The dominant finding is, that these subunits, which we denoted as "elements" for not-fragmented dust particles are essentially within the same size range as the individual "fragments" dispersed by the impact-fragmented dust particles. This observation seems to show that the fragments are not formed by the impact, but pre-existing in the parent dust agglomerate, and simply broken apart during the impact. They may have their own internal substructure of smaller sub-micron sized granules of mixed composition as is known for IDP's. For a discussion of similarities between our particles and IDP's see Langevin et al. (2016).

Among the overwhelming wealth of the collected samples there are a few cases of dust particles $>100$ $\mu \mathrm{m}$ that do not show any damage and present only little substructure at the level of our image resolution. They might either belong to the lower part of the speed population or possess higher density and higher strength. As we cannot measure the speed of the incoming dust particles we use the speed values measured by GIADA (Rotundi et al., 2015, Della Corte et al. 2015). In the parallel paper of Merouane et al. (2016) it is shown, that several cases of big impact events seem to correspond to much larger dust particles fragmenting e.g. at the walls of the entry funnel of the instrument (Kissel et al., 2009). The largest dust particles $(>100 \mu \mathrm{m})$ found on our targets generally show disruption upon impact, which implies that these particles have a lower density, a higher porosity and a weaker mechanical strength compared to smaller particles. This is in agreement with laboratory measurements of dust agglomerates and with models that predict a reduction of both, the value of the tensile strength, and of the speed threshold for disruption with increasing agglomerate size (Weidling et al. 2012, Meru et al 2013, Skorov et al. 2012).

To get a hand on the values for the strength, we use very simple phenomenological concepts, which are more a dimensional analysis than an explicit theory. We wanted to stay as close as possible to the data, and use a minimum of theoretical modelling. We were able to obtain an order of magnitude for the mechanical strength. It is in the order of several $1000 \mathrm{~Pa}$ with a validity of about a factor of two. It results mainly from the uncertainties in the estimate of the dust's mass density. For fragmented dust, energy density arguments gave an upper bound for the mechanical strength whereas the analysis of the distribution of the fragment sizes resulted in a direct estimate. For undamaged particles, only a lower bound could be asserted derived from the energy density. This lower bound can rise considerably, when taking into account a size dependent speed (Agarwal et al., 2007).

The values derived here are for agglomerate sizes up to a few $100 \mu \mathrm{m}$ which hit our targets at low speed. However, the inner strength of their constituents, the "elements", might be much higher than that of the entire agglomerate. This may be due to a sticking enhancement by refractory organic material (Kudo 2002). Some substructures of IDP's are of this size and an organic glue has been found down to nanometer scales (Flynn 2013, Matrajt et al., 2012). As was previously reported (Langevin et al., 2016), many of the particles observed by COSIMA are dark, although they appear bright before the background of the gold black. The reason might be the presence of organics. Our observation of the relative stability of fragment sizes of some tens of micrometers supports the argument in this direction.

Most of the dust particles collected by COSIMA are thought to have lost their volatiles, i.e. they are the remaining mineral and refractory organic components only. According to Blum et al. 2014, Gundlach et al.2014, Aumatell and Wurm 2013 the presence of frozen volatiles increases the strength values by roughly one order of magnitude.

For dust sizes much larger than a few $100 \mu \mathrm{m}$, the physics of interaction may change. It may no longer be described by adhesion forces only (Lee et al., 1995). For very large objects, the cometnuclei, there was a "fragmentation experiment" conducted by nature itself, when Shoemaker-Levy 9 was disrupted during its approach to Jupiter in 1992. Scotti and Melosh, (1993) estimated differential gravitational forces from which strength values can be derived to be in the order of $\sim 10 \mathrm{~Pa}$. Housen and Holsapple (1990) have made an effort to connect data between those enormously different scale lengths by establishing a general scaling law for strength by dimensional analysis. They include in 


\section{ACCEPTED MANUSCRIPT}

their work also previous findings, that breakup not only depends on the energy density transmitted to the objects, but also on the size of the object and the strain rate experienced. For strength dominated objects, (sizes less than a few $\mathrm{km}$ ) they use an approximate scaling law for the radius dependence: $\sigma \sim r^{-\propto}, 0.24<\propto<0.5$ ( cf. Benz and Asphaug 1994). When taking our value of $10^{3} \mathrm{~Pa}$ (and after correction for the presence of volatiles $10^{4} \mathrm{~Pa}$ ) at $100 \mu \mathrm{m}$ size and the Shoemaker-Levy 9 value of $\sim 10 \mathrm{~Pa}$ at $\mathrm{km}$ size $\sigma \approx 200 \cdot r^{-0.43}$ Pa follows (for $r$ in meters).

The present observations point to weak mechanical strength as a possible, general feature of cometary matter. This is fully consistent with the very low strength (10 to $40 \mathrm{~Pa})$ observed by OSIRIS at much larger scales (several $10 \mathrm{~m}$ through the break-up of 'overhangs', Groussin et al., 2015). Our observation of stable elements having sizes of a few tens of micrometers may be of significance. This can be a hint for a size dependence of the binding physics which in turn could correspond to different phases of the accretion process.

\section{Acknowledgments:}

COSIMA was built by a consortium led by the Max-Planck-Institut für Extraterrestrische Physik, Garching, Germany in collaboration with Laboratoire de Physique et Chimie de l'Environnement et de l'Espace, Orléans, France, Institut d'Astrophysique Spatiale, CNRS/Université Paris Sud, Orsay, France, Finnish Meteorological Institute, Helsinki, Finland, Universität Wuppertal, Wuppertal, Germany, von Hoerner und Sulger GmbH, Schwetzingen, Germany, Universität der Bundeswehr, Neubiberg, Germany, Institut für Physik, Forschungszentrum Seibersdorf, Seibersdorf, Austria, Institut für Weltraumforschung, Österreichische Akademie der Wissenschaften, Graz, Austria and is led by the Max-Planck-Institut für Sonnensystemforschung, Göttingen, Germany. The support of the national funding agencies of Germany (DLR, grant 50 QP 1302), France (CNES), Austria, Finland and the ESA Technical Directorate is gratefully acknowledged. One of the co-authors is supported by the Italian Space Agency within the ASI-INAF agreements I/032/05/0 and I/024/12/0. We thank the 


\section{ACCEPTED MANUSCRIPT}

Rosetta Science Ground Segment at ESAC, the Rosetta Mission Operations Centre at ESOC and the Rosetta Project at ESTEC for their outstanding work enabling the science return of the Rosetta Mission. We thank the referees for having greatly improved the manuscript.

\section{References:}

Agarwal J., Mueller M., Gruen E., 2007. Dust Environment Modelling of Comet 67P/Churyumov-Gerasimenko. Space Sci Rev 128, 79-131.

Aumatell G., Wurm G., 2014. Ice aggregate contacts at the nm-scale. Mon. Not. R. Astronom Soc. 437 (1): 690-702. Doi: 10.1093/mnras/stt1921.

Beitz E., Güttler C., Blum J., Meisner T., Teiser J., Wurm G., 2011. Low-velocity collisions of centimeter-sized dust agglomerates. ApJ, 736:34. doi:10.1088/0004-637X/736/1/34

Bentley M. S., Torkar K., Romstedt J., 2014. The structure of cometary dust - first results from the MIDAS Atomic Force Microscope onboard ROSETTA. AGU Fall Meeting San Francisco, paper P32B-03.

Benz W. and Asphaug E., 1994. Shoemaker-Levy 9 and the Tidal Disruption of Comets, LPSC XXV, p.101-102.

Blum J. and Wurm G., 2008. The Growth Mechanisms of Macroscopic Bodies in Protoplanetary Disks. Annu. Rev. Astro. Astrophys., vol. 46, no. 1, pp. 21-56.

Blum J., Gundlach B., Mühle S., Trigo-Rodriguez J.M., 2014. Comets formed in solar-nebula instabilities! - An experimental and modeling attempt to relate the activity of comets to their formation process. Icarus $235,156-169$.

Blum J. and Schräpler R., 2004. Structure and Mechanical Properties of High-Porosity Macroscopic Agglomerates Formed by Random Ballistic Deposition,

Phys. Rev. Letters, 93, 11.

Blum J., Schräpler R., Davidsson B.J.R., Trigo-Rodriguez J.M., 2006. The Physics of Protoplanetesimal Dust Agglomerates. I. Mechanical Properties and Relations to Primitive Bodies in the Solar System. ApJ, 652:1768-1781.

Bower A.F., 2010. Applied Mechanics of Solids, C\&C Press, Taylor and Francis.

Bradley J.P., 2013. How and where did GEMS form? Geochimica et Cosmochimica Acta 107, 336340 .

Brownlee D., 1985. Cosmic dust - Collection and research. Annu. Rev. Earth Planet. Sci., 13, 147173.

Brownlee D., Joswiak D., Matrajt, 2012. Overview of the rocky component of Wild 2 comet samples: Insight into the early solar system, relationship with meteoritic materials and the differences between comets and asteroids. Meteoritics \& Planetary Science 47, Nr 4, 453-470 (2012). doi: 10.1111/j.19455100.2012.01339.x. 


\section{ACCEPTED MANUSCRIPT}

Brownlee D., 2014. The Stardust Mission: Analyzing Samples from the Edge of the Solar System. Annu. Rev. Earth Planet. Sci. 42:179-205.

Ceplecha Z. and McCrosky R.E., 1976. Fireball end heights - A diagnostic for the structure of meteoric material. J. Geophys. Res. 81, 6257-6275.

Clark B. C., Green S.F., Economou T.E., Sandford S.A., Zolensky M.E., McBride N., Brownlee D., 2004. Release and fragmentation of aggregates to produce heterogeneous, lumpy coma streams. J. Geophys. Res. 109, E12S03, doi: 10.1029/2004JE002319.

Della Corte V., Rotundi A. Fulle M., Gruen E., Weissman P., Sordini R., Ferrari M., Ivanovski S., Lucarelli F., Accolla M., Zakharov V., Mazzotta Epifani E., Lopez-Moreno J.J., Rodriguez J., Colangeli L., Palumbo P., Bussoletti E., Crifo J.F., Esposito F., Green S.F., Lamy P.L., McDonnell J.A.M., Mennella V., Molina A., Morales R., Moreno F., Ortiz J.L., Palomba E., Perrin J.M., Rietmeijer F.J.M., Rodrigo R., Zarnecki J.C., Cosi M., Giovane F., Gustafson B., Herranz M.L., Jeronim J.. Leese M.R., Lopez-Jimenez A.C., Altobelli N., 2015. GIADA: shining a light on the monitoring of the comet dust production from the nucleus of 67P/Churyumov Gerasimenko. Astronomy \& Astrophysics manuscript no. aa26208-15 c ESO 2015 August 19, 2015.

Duprat J., Engrand C., Maurette M., Kurat G., Gounelle M., Hammer C., 2007. Micrometeorites from Central Antarctic snow: The CONCORDIA collection.

Advances in Space Research 39, 605-611.

Engrand C., Benzerara K., Leroux H., Duprat J., Dartois E., Bardin N., Delauche L., 2015.

Carbonaceous phases and mineralogy of Ultracarbonaceous Antarctic Micrometeorites identified by C- and N-XANES/STXM and TEM, 46 Lunar and Planetary Science Conference, Paper No. 1902.

Flynn G.J., Wirick S., 2, Keller L.P., 2013. Organic grain coatings in primitive interplanetary dust particles: Implications for grain sticking in the Solar Nebula. Earth Planets Space, 65, 1159-1166.

Fulle M., Della Corte V., Rotundi A., Weissman, P., Juhasz, A., Szego, K., Sordini, R., Ferrari M., Ivanovski S., Lucarelli F., Accolla M., Merouane S., Zakharov V., Mazzotta Epifani E., LopezMoreno J.J., Rodriguez J., Colangeli L., Palumbo P., Gr un E., Hilchenbach M., Bussoletti E., Esposito F., Green S. F., Lamy P. L., McDonnell J. A. M., Mennella V., Molina A., Morales R., Moreno F., Ortiz J. L., Palomba E., Rodrigo R., Zarnecki, J. C., Cosi J. M., Leese M. R., LopezJimenez A. C., Altobelli N., 2015. Density and Charge of Pristine Fluffy Particles from Comet 67P/Churyumov-Gerasimenko. Astrophysical Journal Letters, 802(1): L12 (5pp).

Greenberg, J. M. and Hage J. I., 1990. From interstellar dust to comets - a unification of observational constraints. Ap. J. 361, 260-274.

Greenberg J. M., Li A., 1999. Morphological Structure and Chemical Composition of Cometary Nuclei and Dust. Space Science Reviews 90: 149-161.

Groussin O., Jorda L., Auger A-T. et al., 2015. Gravitational slopes, geomorphology and material strengths of the nucleus of comet $67 \mathrm{P} /$ Churyumov-Gerasimenko from OSIRIS observations. http://dx.doi.org/10.1051/0004-6361/201526379

Güttler C., Krause R., Geretshauser R., Speith R., Blum J., 2009. The Physics of Protoplanetesimal Dust Agglomerates. IV. Toward a Dynamical Model, The Astrophysical Journal 701,130-141. doi:10.1088/0004-637X/701/1/130

Gundlach B. and Blum J., 2014. The stickiness of micrometer sized water-ice particles, The Astrophysical Journal 798, 1. http://dx.doi.org/10.1088/0004-37X/798/1/34. 


\section{ACCEPTED MANUSCRIPT}

Hartmann W.K. 1978, Planet Formation: Mechanism of Early Growth, Icarus 33, 50-61.

Hilchenbach M., et al., 2016. Comet 67P/Churyumov-Gerasimenko - Close-Up on Dust Particle Fragments. ApJLetters 816,2.

Hornung K., Kissel J., Fischer H., Mellado E.M., Kulikov O., Hilchenbach M., Krüger H., Engrand C., Langevin Y., Rossi M., Krueger F.R., 2014. Collecting cometary dust particles on metal blacks with the COSIMA instrument onboard ROSETTA. Planetary and Space Science 103, 309-317.

Hornung K. and Kissel J., 1994. On shock wave ionization of dust particles, Astron. Astrophys. 291,324-336.

Housen K.R. and Holsapple K.A., 1990. On the fragmentation of asteroids and planetary satellites, ICARUS 84, 226-253.

Israelachvili J.N., 2011.Intermolecular and Surface Forces, Academic Press, $3^{\text {rd }}$. Edition.

Kimura, H., Wada, K., Senshu, H. and Kobayashi, H., 2015. Cohesion of Amorphous Silica spheres: Toward a better understanding of the coagulation growth of Silicate dust aggregates, ApJ, 812(1), 67, doi:10.1088/0004-637X/812/1/67.

Kirchner H., Michot G., Schweizer J., 2002. Fracture toughness of snow in shear and tension, Scripta Materialia, 46, 6, 425-429.

Kissel J., Glasmachers A., Grün E., Henkel H., Höfer H., Haerendel G., v.Hoerner H., Hornung K., Jessberger E.K., Krueger F.R., Möhlmann D., Greenberg J.M., Langevin Y., Silen J., Brownlee D., Clark B.C., Hanner M.S., Hoerz F., Sandford S., Sekanina Z., Tsou P., Utterback N.G., Zolensky M.E., Heiss C., 2003. Cometary and Interstellar Dust Analyzer for comet Wild 2. Journal of Geophysical Research 108, E10, 8114.

Kissel J., COSIMA Team, 2009. COSIMA: High resolution time-of-flight secondary ion mass spectrometer for the analysis of cometary dust particles onboard ROSETTA. In:Schulz R.,Alexander C.,Boenhardt H.,Glassmeier K.H.(Eds.), ESA's Mission to the Origin of the Solar System. Springer,NewYork, 201-242.

Kissel J., Krueger F.R., 1987. The Organic Component in Dust from Comet Halley as Measured by the PUMA Mass Spectrometer on Board VEGA 1.\ Nature 326, 755-760.

Kudo T., Kouchi A., Arakawa M., Nakano H., 2002. The role of sticky interstellar organic material in the formation of asteroids. Meteoritics \& Planetary Science 37,1975-1983.

Langevin Y., Hilchenbach M., Ligier N., Merouane S., Hornung K., Engrand C., Schulz R., Kissel J., Ryno J. and the COSIMA team, 2016. Typology of dust particles collected by the COSIMA mass spectrometer in the inner coma of 67P/Churyumov Gerasimenko. Icarus in press: doi: 10.1016/j.icarus.2016.01.027

Lee L.H., 1995. Adhesion and cohesion mechanics of lunar dust on the moon's surface. J. of Adhesion Science and Technology 9, 8, 1103-1124.

Maas D., Krueger F.R., Kissel J., 1990. Mass and Density of Silicate- and CHON-Type Dust Particles Released by Comet P/Halley. Asteroids, Comets, Meteors III, Proceedings of a meeting (AMC 89) held at the Astronomical Observatoy of the Uppsala University, Ed. C.I. Lagerquist, H. Rickman B.A: Lindblad, p. 389 ff. 


\section{ACCEPTED MANUSCRIPT}

Matrajt G., Messenger S., Brownlee D., Joswiak, D., 2012. Diverse forms of primordial organic matter identified in interplanetary dust particles. Meteoritics \& Planetary Science 47, 4, 525-549. doi: $10.1111 / \mathrm{j} .1945-5100.2011 .01310 . x$

Merouane S., Langevin Y., Stenzel O., Altobelli N., Della Corte V., Fischer H., Fulle M., Hornung K., Silen J., Ligier N., Rotundi A., Ryno J., Schulz R., Hilchenbach M., Kissel J., and the COSIMA Team, 2016. Dust particles flux and size distribution in the coma of 67P/Churyumov-Gerasimenko measured in-situ by the COSIMA instrument on board Rosetta. Astronomy \& Astrophysics manuscript no. draft flux $2015^{\circ} 12^{*}$ 08, Submitted Dec 9, 2015.

Meru F., Geretshauser R.J., Schaefer Ch., Speith R., Kley W., 2013.

Growth and fragmentation of centimetre-sized dust aggregates: the dependence on aggregate size and porosity. Mon. Not. R. Astronom. Soc. 435, p.2371.

Mukai T., Ishimoto H., Kozasa T., Blum J., Greenberg J.M., 1992. Radiation pressure forces of fluffy porous grains, Astron. Astrophys. 262, 315-320.

Pätzold M., Andert T., Hahn M., Asmar S.W., Barriot J.-P., Bird M.K., Häusler B., Peter K., Tellmann S., Grün E., Weissman P.R., Sierks H., Jorda L., Gaskell R., Preusker F., Scholten F., 2016. A homogeneous nucleus for comet $67 \mathrm{P} /$ Churyumov-Gerasimenko from its gravity field. Nature 530, 63-65. doi:10.1038/nature16535.

Paquette, J.A., Engrand C., Stenzel O., Hilchenbach M., Kissel J., and the COSIMA Team, 2016. Searching for calcium-aluminum-rich inclusions in cometary particles with Rosetta/COSIMA. Submitted to Meteoritics and Planetary Science.

Riedler W. et al., 2007. MIDAS The Micro-Imaging Dust Analysis System for the Rosetta Mission. Space Sci. Rev. 128, 869-904.

Rotundi A. et al., 2015. Dust measurements in the coma of comet 67P/Churyumov-Gerasimanko inbound to the Sun. Science 347, Issue 6220, aaa3905.

Rumpf, H. 1958. Grundlagen und Methoden des Granulierens. Chem. Ing. Tech. 30, 144-158.

Reynolds G.K., Fu Y.S., Cheong M.J., Hounslow M.J., Salman A.D., 2005. Breakage in granulation: A review. Chem. Eng. Science 60,3969-3992.

Sánchez, P. and Scheeres, D. J., 2014. The strength of regolith and rubble pile asteroids. Meteoritics \& Planetary Science, 49: 788-811. doi: 10.1111/maps.12293.

Seizinger A. et al. 2012. Compression behavior of porous dust agglomerates. Astron. \&Astrophys. 541, A59.

Seizinger A., Speith R., Kley W., 2013. Tensile \& shear strength of porous dust agglomerates. Astron. \& Astrophys. 559, id.A19. doi: $\underline{10.1051 / 0004-6361 / 201322046}$

Schulz R., Hilchenbach M., Langevin Y., Kissel J., Silen J., Briois Ch., Engrand C., Hornung K., Baklouti D., Bardyn A., Cottin H., Fischer H., Fray N., Godard M., Lehto H., Le Roy L., Merouane S., Orthous-Daunay F.-R., Paquette J., Rynö J., Siljeström S., Stenzel O., Thirkell L., Varmuza K., Zaprudin B., 2015. Comet 67P/Churyumov-Gerasimenko sheds dust coat accumulated over the past four years. Nature 518, 216-218. doi:10.1038/nature14159.

Skorov Yu. and Blum J., 2012, Dust release and tensile strength of the non-volatile layer of cometary nuclei. Icarus 221,(1),1-11.

Scotti J.V., Melosh H.J., 1993. Estimate of the size of comet Shoemaker-Levy 9 


\section{ACCEPTED MANUSCRIPT}

from a tidal breakup model. Nature 365, 6,733-735.

Setoh M., Nakamura A.M., Hirata N., Hiraoka K., Masahiko A., 2007. Collisional disruption of weakly sintered porous targets at low-impact velocities. Earth Planets Space, 59, 319-324.

Thomson R.M., 1973. The fracture crack as an imperfection in a nearly perfect solid, Annual Review of Materials Science, 3, 31-51.

Weidling R., Güttler C., Blum J., 2012. Free Collisions in a Microgravity Many-Particle Experiment. I. Dust Aggregate Sticking at Low Velocities. Icarus, 218, 688-700.

Wurm G., Paraskov G., Krauss O., 2005. Ejection of dust by elastic waves in collisions between millimeter- and centimeter-sized dust aggregates at 16.5 to $37.5 \mathrm{~m} / \mathrm{s}$ impact velocities. Phys. Rev. E $71,021304$.

\section{Highlights}

- We collected dust in the near coma of Comet 67P/Churyumov-Gerasimenko.

- Dust particles fragment upon impact on our collection plates already at speeds of a few $\mathrm{m} / \mathrm{s}$.

- The dust seems to consist of elements of a few tens of micrometers in size which are loosely bound together.

- An order of magnitude for the strength is derived $\left(\approx 10^{3}\right.$ Pa for fragmenting dust of few hundred micrometers size). 
Graphical abstract: 


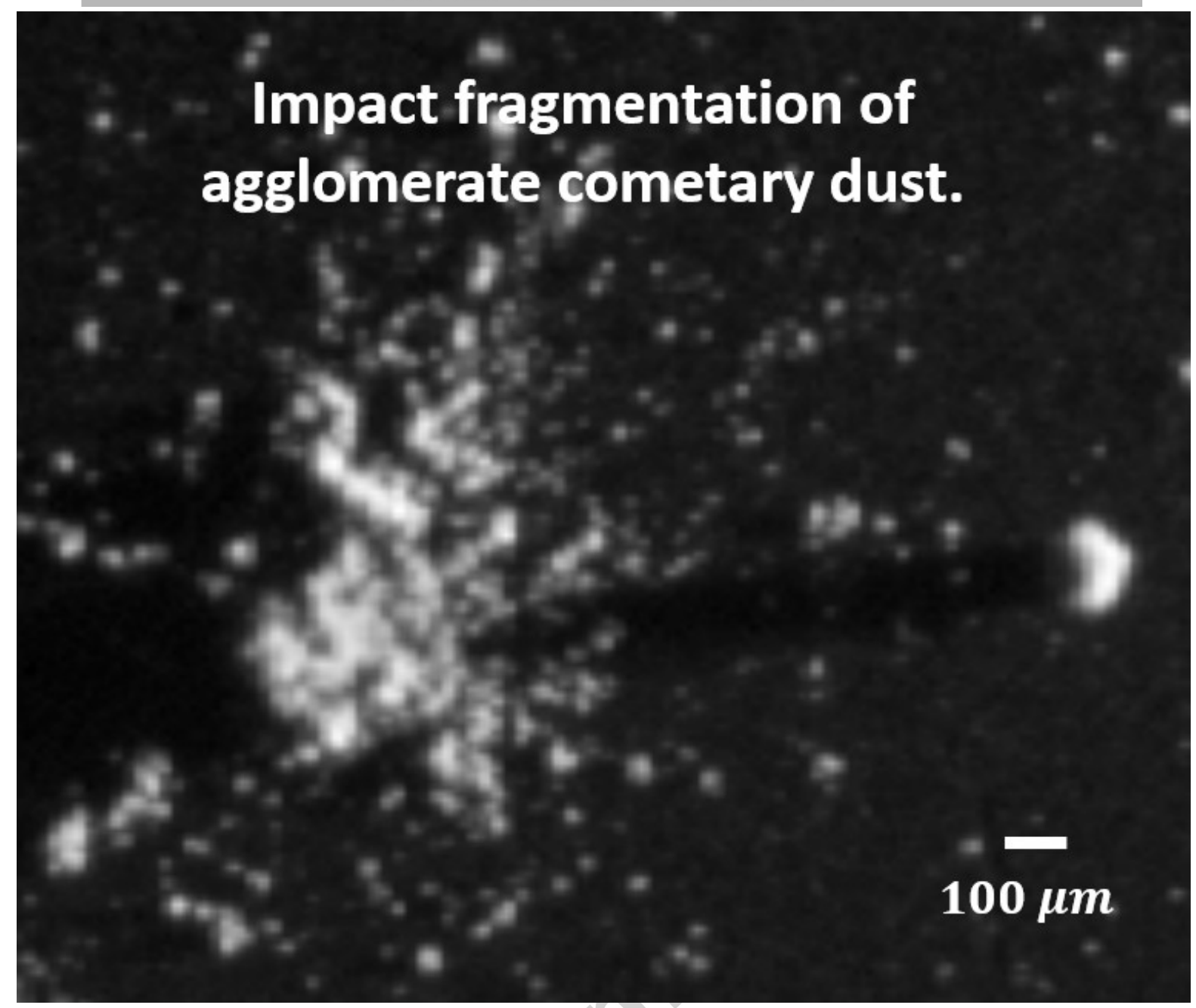

